\title{
Temporal and spatial Lagrangean decompositions in multi-site, multi-period production planning problems with sequence-dependent changeovers
}

\author{
Sebastian Terrazas-Moreno $^{\mathrm{a}}$, Philipp Trotter ${ }^{\mathrm{b}}$, Ignacio E. Grossmann ${ }^{\mathrm{a}^{*}}$ \\ ${ }^{a}$ Carnegie Mellon University, 5000 Forbes Ave, Pittsburgh PA 15232, USA, grossmann@cmu.edu \\ ${ }^{b}$ RWTH Aachen University, Templergraben 55,52056 Aachen, Germany,philipp.trotter@rwth-aachen.de
}

\begin{abstract}
We address in this paper the optimization of a multi-site, multi-period, and multi-product planning problem with sequence-dependent changeovers, which is modeled as a mixedinteger linear programming (MILP) problem. Industrial instances of this problem require the planning of a number of production and distribution sites over a time span of several months. Temporal and spatial Lagrangean decomposition schemes can be useful for solving these types of large-scale production planning problems. In this paper we present a theoretical result on the relative size of the duality gap of the two decomposition alternatives. We also propose a methodology for exploiting the economic interpretation of the Lagrange multipliers to speed the convergence of numerical algorithms for solving the temporal and spatial Lagrangean duals. The proposed methods are applied to the multi-site multi-period planning problem in order to illustrate their computational effectiveness.
\end{abstract}

Keywords: Lagrangean decomposition, production planning, temporal decomposition, spatial decomposition.

\section{Introduction}

The optimal planning of a network of manufacturing sites and markets is a complex problem. It involves assigning which products to manufacture in each site, how much to ship to each market and how much to keep in inventory to satisfy future demand. Each site has different production capacities and operating costs, while demand for products varies significantly across markets. Production and distribution planning is concerned with mid to long-term decisions usually involving several months, adding a temporal dimension to the spatial distribution given by the multi-site network. The production of

* Author to whom correspondence should be addressed. E-mail: grossmann@cmu.edu 
each product can involve a setup or cleaning time that in some cases is sequencedependent. When setups and sequence-dependent transitions are included, the optimal planning problem becomes a mixed-integer linear programming (MILP) problem. The computational expense of solving large-scale MILP problems can be decreased by using decomposition techniques. This paper presents temporal and spatial Lagrangean decompositions that allow the independent solution of time periods, production sites, and markets. The importance of choosing between alternative Lagrangean relaxations of the same planning model is discussed by Gupta and Maranas (1999). Jackson and Grossmann (2003) use temporal decomposition for solving a multi-site, multi-period planning problem. They report that temporal decomposition provides a tighter bound on the full space solution and has faster dual convergence than spatial decomposition. Wu and Ierapetritou (2006) also implement Lagrangaen decomposition on a multi-period scheduling problem. These authors propose to use the Nelder-Mead approach as an alternative to subgradient method for updating the multipliers. Temporal decomposition using this approach results in a significant reduction in computational time. Neiro and Pinto (2006) use temporal Lagrangean decomposition to solve a multi-period MINLP planning problem under uncertainty concerning a petroleum refinery. They find that this decomposition scheme helps overcome the exponential increase in solution time that occurs with the full space model. In a problem similar to the one presented in this paper, Chen and Pinto (2008) use Lagrangean-based decomposition techniques for solving the temporal decomposition of a continuous flexible process network. They use subgradient methods to solve the decomposed problem and find that temporal decomposition strategies result in a reduction of computational time of several orders of magnitude.

From the papers mentioned above, it is evident that temporal decomposition is an efficient solution approach for multi-period planning problems. It has been found to provide a tighter bound on the optimal solution and to have better convergence properties than Lagrangean spatial decomposition in mulit-site problems. However, there is no rigorous proof and generalization of the observed result. One objective of this paper is to compare the bounds obtained through Lagrangean temporal and spatial decompositions for a class of MILP problems derived from the lot-sizing problem with setup and sequence-dependent changeover times (Pochet and Wolsey, 2006). The second objective 
is to use the economic interpretation of the Lagrange multipliers to provide a reduced dual search space and accelerate the convergence of the optimal multipliers.

This paper is organized as follows. Section 2 presents the details of the MILP formulation for the production planning problem. Section 3 describes the implementation of temporal and spatial decomposition of the MILP problem in section 2. In part 4 we review some important theoretical concepts and in section 5 we introduce a result where the dual gap of temporal decomposition is found to be at least as small as the dual gap for spatial decomposition. Sections 6 and 7 contain our novel approach for exploiting the economic interpretation of the Lagrange multipliers to reduce the search space for the optimal multipliers. Section 8 presents four numerical examples of increasing size and complexity for the multi-site multi-period planning problem where the theoretical result is confirmed, and where the economic interpretation of the multipliers is used to speed the convergence of numerical algorithms for solving the decomposed problem. Finally, section 9 presents our conclusions and ideas for future work.

\section{Problem Statement}

Given is a set of products that are manufactured in several continuous multi-product production sites and shipped to a set of markets where they are sold. Let $I, S$, and $M$ be the sets of products, production sites, and markets, respectively. Figure 1 shows the multi-period, multi-site network structure.

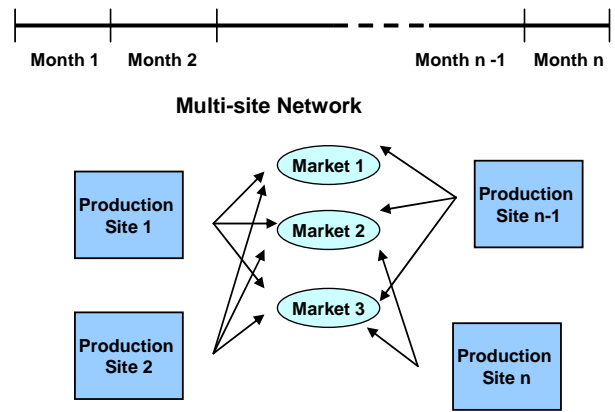

Fig 1. Network of production sites and markets for a multi-period planning problem 
There is a finite time horizon divided into time periods of length $L_{t}$. The set of time periods is denoted by $T$. Given is also a forecast of the demand of each product in each market at the end of the time periods. The problem is to determine the production in each site, the inventory levels, and the amounts of products shipped to each market during each time period in order to maximize the profit. We assume that the size of the problem may prohibit its direct solution, and we consider that temporal and spatial Lagrangean decomposition techniques are alternatives to overcome this challenge. One objective of this work is to rigorously compare the strength of the Lagrangean duals resulting from each decomposition scheme. Another objective is to illustrate how the economic interpretation of the Lagrange multipliers of the constraints that are relaxed in both decompositions can be used to reduce the dual search space.

The following mixed-integer linear programming (MILP) model, which is formulated in a generic way, corresponds to the profit maximization planning problem described above.

$\max \pi=\sum_{t \in T} \sum_{s \in S} \sum_{s \in M} \beta_{t}^{m} s l_{t}^{s, m}-\sum_{t \in T} \sum_{s \in S}\left(\alpha_{t}^{s} x_{t}^{s}+\delta_{t}^{s} v_{t}^{s}+T C_{t}^{s} t r n_{t}^{s}\right)-\sum_{t \in T} \sum_{s \in S} \sum_{m \in M} \gamma_{t}^{s, m} f_{t}^{s, m}$

s.t.

$$
\begin{array}{ll}
v_{t-1}^{s}+x_{t}^{s}=\sum_{m \in M} f_{t}^{s, m}+v_{t}^{s} & \forall t \in T, s \in S \\
x_{t}^{s} \leq x_{s}^{U B} s t p_{t}^{s} & \forall t \in T, s \in S \\
a_{t}^{s} x_{t}^{s}+b s_{t}^{s} s t p_{t}^{s}+b t_{t}^{s} t r n_{t}^{s} \leq L_{t} & \forall t \in T, s \in S \\
C s_{t}^{s}\left(s t p_{t}^{s}\right)+C t_{t}^{s}\left(t r n_{t}^{s}\right)+C t_{t}^{\prime s}\left(t r n_{t+1}^{s}\right) \leq e_{t}^{s} & \forall t \in T, s \in S \\
f_{t}^{s, m}=s l_{t}^{s, m} & \forall t \in T, s \in S, m \in M \\
\sum_{s \in S} s l_{t}^{s, m} \leq d_{t}^{m} & \forall t \in T, m \in M \\
x_{t}^{s} \leq x_{s}^{U B} ; v_{t}^{s} \leq v_{s}^{U B} & \forall t \in T, s \in S \\
f_{t}^{s, m} \leq f_{s, m}^{U B} & \forall t \in T, s \in S, m \in M \\
x_{t}^{s} \in \mathfrak{R}_{+}^{|I|}, v_{t}^{s} \in \mathfrak{R}_{+}^{|I|}, t r n_{t}^{s} \in \mathfrak{R}_{+}^{|I| \times|I| \times \# t r n \text { variables }} & \forall t \in T, s \in S \\
f_{t}^{s, m} \in \mathfrak{R}_{+}^{|I|}, s l_{t}^{s, m} \in \mathfrak{R}_{+}^{|I|} & \forall t \in T, s \in S, m \in M \\
s t p_{t}^{s} \in\{0,1\}^{|I|} & \forall t \in T, s \in S \\
0 \leq t r n_{t}^{s} \leq 1 & \forall t \in T, s \in S
\end{array}
$$


Equation (1.1) represents the maximization of profit, computed as sales minus production, inventory, transition, and transportation costs. The coefficients $\beta_{t}^{m}, \alpha_{t}^{s}, \delta_{t}^{s}, T C_{t}^{s}$, and $\gamma_{t}^{s, m}$ are row vectors of length $|I|$, where $I$ is the set of products produced and sold in the multi-site network. Equation (1.2) is the production site mass balance; $x_{t}^{s}$ represents production, $v_{t}^{s}$ inventory levels, and $f_{t}^{s, m}$ amount of product shipped from $s$ to $m$. Constraint (1.3) enforces the condition that a product can only be produced if there is a setup assigned to it $\left(s t p_{s}^{t}\right)$. Constraint (1.4) limits the time balance at each production site involving the transition variables $\operatorname{trn}_{s}^{t}$. The row vector $a_{t}^{s}$ contains the inverse of the production rates of all products, while $b s_{t}^{s}$ and $b t_{t}^{s}$ are vectors with set up and transition times. $L_{t}$ is the duration of time period $t$. Constraint (1.5) is a compact representation of a set of general sequencing constraints. In general, assume there are $k=1, \ldots, K$ constraints in this set. Then $C s_{t}^{s}$ is a matrix with $K$ rows and $|I|$ columns, and $C t_{t}^{s}$ and $C t_{t}^{\prime s}$ are matrices with the same number of rows and $|I| \times|I| \times(\#$ trn variables) columns. The expression \# trn variables represents the different types of transition variables used in the model, for instance, transition within time periods, transitions across time periods, etc. The vector of right hand side coefficients $e_{t}^{s}$ has dimension $K$. Equation (1.6) expresses the condition that all products that arrive to a market are sold. The vector $d_{t}^{m}$ contains the market demands for all the products at each time period. The rest of the constraints involve the upper bounds, ranges, and integrality conditions of the decision variables. It is important to note that the transition variables $\operatorname{trn}_{s}^{t}$ are continuous and bounded between zero and one. We assume that constraint (1.5) contains sequencing constraints of the type shown in Appendix A and proposed by Erdirik-Dogan and Grossmann (2008), where transition variables always take values of 0 or 1 , even if they are declared as continuous.

The explicit model used for the examples included in this paper is shown in Appendix A. 


\section{Temporal and spatial Lagrangean decompositions.}

We assume that large-scale instances of the planning problem given by equations (1.1) (1.13) cannot be solved in full space, and we consider that temporal and spatial Lagrangean decomposition techniques are alternatives to overcome this challenge. In this section we describe both ways of decomposing problem (1).

\section{Temporal decomposition}

Problem (1) is a multi-period problem where the solution of different time periods is linked by the presence of inventory $v_{t}^{s}$ and by production sequencing across time periods, as modeled by some of the variables in the vector $t r n_{t}^{s}$. We use Lagrangean decomposition for decoupling the solution across time periods. First we introduce the copy variables $\bar{v}_{t}^{s}$ and $\overline{t r n}_{t}^{s}$ in constraints (1.2) and (1.5) along with $\bar{v}_{t}^{s}=v_{t}^{s}$ and $\overline{t r n}_{t}^{s}=t r n_{t}^{s}$. We rewrite problem (1) including these changes:

$$
\begin{array}{ll}
\max \pi=\sum_{t \in T} \sum_{s \in S m \in M} \sum_{t} \beta_{s}^{m} l_{t}^{s, m}-\sum_{t \in T} \sum_{s \in S}\left(\alpha_{t}^{s} x_{t}^{s}+\delta_{t}^{s} v_{t}^{s}+T C_{t}^{s} t r n_{t}^{s}\right)-\sum_{t \in T} \sum_{s \in S} \sum_{m \in M} \gamma_{t}^{s, m} f_{t}^{s, m} \\
\text { s.t. } & \forall t \in T, s \in S \\
-s & \\
v_{t-1}+x_{t}^{s}=\sum_{m \in M} f_{t}^{s, m}+v_{t}^{s} & \forall t \in T, s \in S \\
x_{t}^{s} \leq x_{s}^{U B} s t p_{t}^{s} & \forall t \in T, s \in S \\
a_{t}^{s} x_{t}^{s}+b s_{t}^{s} s t p_{t}^{s}+b t_{t}^{s} t r n_{t}^{s} \leq L_{t} & \forall t \in T, s \in S \\
C s_{t}^{s}\left(s t p_{t}^{s}\right)+C t_{t}^{s}\left(\operatorname{trn}_{t}^{s}\right)+C t_{t}^{\prime s}\left(\overline{\text { trn }_{t+1}}\right) \leq e_{t}^{s} & \forall t \in T, s \in S, m \in M \\
f_{t}^{s, m}=s l_{t}^{s, m} & \forall t \in T, m \in M \\
\sum_{s \in S} s l_{t}^{s, m} \leq d_{t}^{m} & \forall t \in T, s \in S \\
x_{t}^{s} \leq x_{s}^{U B} ; v_{t}^{s} \leq v_{s}^{U B}, v_{t} \leq v_{s}^{U B} & \forall t \in T, s \in S, m \in M \\
f_{t}^{s, m} \leq f_{s, m}^{U B} & \forall t \in T, s \in S \\
x_{t}^{s} \in \mathfrak{R}_{+}^{|I|}, v_{t}^{s} \in \mathfrak{R}_{+}^{|I|}, t r n_{t}^{s} \in \mathfrak{R}_{+}^{|I| \times|I| \times \# t r n \text { variables }} & \forall t \in T, s \in S \\
-s & \forall t \in T, s \in S, m \in M \\
v_{t} \in \mathfrak{R}_{+}^{|I|}, \overline{t r n}_{t}^{s} \in \mathfrak{R}_{+}^{|I| \times|I| \times \# t r n \text { variables }} & \forall t \in T, s \in S \\
f_{t}^{s, m} \in \mathfrak{R}_{+}^{|I|}, s l_{t}^{S, m} \in \mathfrak{R}_{+}^{|I|} & \\
s t p_{t}^{s} \in\{0,1\}^{|I|} &
\end{array}
$$




$$
\begin{array}{lc}
0 \leq \operatorname{trn}_{\mathrm{t}}^{\mathrm{s}} \leq 1,0 \leq \overline{\operatorname{trn}}_{\mathrm{t}}^{\mathrm{s}} \leq 1 & \forall t \in T, s \in S \\
\bar{v}_{t}^{s}=v_{t}^{s} & \forall t \in T, s \in S \\
\overline{\operatorname{trn}}_{t} s=\operatorname{trn}_{t}^{s} & \forall t \in T, s \in S
\end{array}
$$

Temporal Lagrangean relaxation of Problem (1) is as follows:

$$
\begin{aligned}
\max \pi^{t}(\lambda v, \lambda t r)= & \sum_{t \in T} \sum_{s \in S} \sum_{m \in M} \beta_{t}^{m} s l_{t}^{s, m}-\sum_{t \in T} \sum_{s \in S}\left(\alpha_{t}^{s} x_{t}^{s}+\delta_{t}^{s} v_{t}^{s}+T C_{t}^{s} t r n_{t}^{s}\right)- \\
& \sum_{t \in T} \sum_{s \in S} \sum_{m \in M} \gamma_{t}^{s, m} f_{t}^{s, m}+\sum_{t \in T} \sum_{s \in S} \lambda v_{t}^{s}\left(v_{t}^{s}-\bar{v}_{t}^{s}\right)+\sum_{t \in T} \sum_{s \in S} \lambda t r_{t}^{s}\left(t r n_{t}^{s}-\overline{\operatorname{trn}}_{t}^{s}\right)
\end{aligned}
$$

s.t.(1.2b),(1.3),(1.4),(1.5b),(1.6)-(1.13).

Since the time periods are decoupled, each of them can be solved independently yielding a temporal Lagrangean decomposition. We use the problem $\left(T L_{\lambda}\right)$ as written out above in the theoretical analysis presented in the following sections, but we exploit the decomposable structure when carrying out the computations.

\section{Spatial decomposition}

Equation (1.6) links the solution of production sites and markets. Without this constraint the sales at the markets $s l_{t}^{s, m}$ would be independent of the product shipments from production sites $f_{t}^{s, m}$. We introduce the copy variable $\bar{f}$ and the constraint $\bar{f}=f$ in Problem (1) as follows:

$$
\begin{array}{ll}
\max \pi=\sum_{t \in T} \sum_{s \in S} \sum_{m \in M} \beta_{t}^{m} s l_{t}^{s, m}-\sum_{t \in T} \sum_{s \in S}\left(\alpha_{t}^{s} x_{t}^{s}+\delta_{t}^{s} v_{t}^{s}+T C_{t}^{s} t r n_{t}^{s}\right)-\sum_{t \in T} \sum_{s \in S} \sum_{m \in M} \gamma_{t}^{s, m} \bar{f}_{t}^{s, m} \\
\text { s.t. } & \forall t \in T, s \in S \\
v_{t-1}^{s}+x_{t}^{s}=\sum_{m \in M} f_{t}^{s, m}+v_{t}^{s} & \forall t \in T, s \in S \\
x_{t}^{s} \leq x_{s}^{U B} s t p_{t}^{s} & \forall t \in T, s \in S \\
a_{t}^{s} x_{t}^{s}+b s_{t}^{s} s t p_{t}^{s}+b t_{t}^{s} t r n_{t}^{s} \leq L_{t} & \forall t \in T, s \in S \\
C s_{t}^{s}\left(s t p_{t}^{s}\right)+C t_{t}^{s}\left(t r n_{t}^{s}\right)+C t_{t}^{\prime s}\left(t r n_{t+1}^{s}\right) \leq e_{t}^{s} & \forall t \in T, s \in S, m \in M \\
\bar{f}_{t}^{s, m}=s l_{t}^{s, m} &
\end{array}
$$




$$
\begin{array}{ll}
\sum_{s \in S} s l_{t}^{s, m} \leq d_{t}^{m} & \forall t \in T, m \in M \\
x_{t}^{s} \leq x_{s}^{U B} ; v_{t}^{s} \leq v_{s}^{U B} & \forall t \in T, s \in S \\
f_{t}^{s, m} \leq f_{s, m}^{U B}, \bar{f}_{t}^{s, m} \leq f_{s, m}^{U B} & \forall t \in T, s \in S, m \in M \\
x_{t}^{s} \in \mathfrak{R}_{+}^{|I|}, v_{t}^{s} \in \mathfrak{R}_{+}^{|I|}, t^{s} n_{t}^{s} \in \mathfrak{R}_{+}^{|I| \times|I| \times \# t r n \text { variables }} & \forall t \in T, s \in S \\
f_{t}^{s, m} \in \mathfrak{R}_{+}^{|I|}, \bar{f}_{t}^{s, m} \in \mathfrak{R}_{+}^{|I|}, s l_{t}^{s, m} \in \mathfrak{R}_{+}^{|I|} & \forall t \in T, s \in S, m \in M \\
s t p_{t}^{s} \in\{0,1\}^{|I|} & \forall t \in T, s \in S \\
0 \leq t r n_{t}^{s} \leq 1 & \forall t \in T, s \in S \\
\bar{f}_{t}^{s, m}=f_{t}^{s, m} & \forall t \in T, s \in S
\end{array}
$$

By relaxing constraint (1.16) the spatial Lagrangean relaxation is as follows:

$$
\begin{aligned}
\max \pi^{s}(\lambda f) & =\sum_{t \in T} \sum_{s \in S} \sum_{m \in M} \beta_{t}^{m} s l_{t}^{s, m}-\sum_{t \in T} \sum_{s \in S}\left(\alpha_{t}^{s} x_{t}^{s}+\delta_{t}^{s} v_{t}^{s}+T C_{t}^{s} t r n_{t}^{s}\right) \\
& -\sum_{t \in T} \sum_{s \in S} \sum_{m \in M} \gamma_{t}^{s, m} \bar{f}_{t}^{s, m}+\sum_{t \in T} \sum_{s \in S} \lambda f_{t}^{s, m}\left(f_{t}^{s, m}-\bar{f}_{t}^{s, m}\right)
\end{aligned}
$$

s.t. (1.2) -(1.6b),(1.7) -(1.13).

We should note that the spatial Lagrangean decomposition $\left(S L_{\lambda}\right)$ can be solved independently for markets and production sites. Once more, we keep the problem $\left(S L_{\lambda}\right)$ as above in the theoretical analysis, but we exploit the decomposable structure when carrying out the computations.

It has been reported in previous work (Jackson \& Grossmann, 2003) that temporal decomposition schemes in certain types of planning problems are superior to spatial ones in terms of required computational effort to find a good solution, as well as in terms of the magnitude of the duality gap. To the best of our knowledge there is no rigorous result that explains this behavior. Next, we review some important concepts and then present a theoretical result that compares the strength of temporal and spatial duals. 


\section{Background}

We present a brief overview of some concepts related with Lagrangean relaxation that will be used in the next section. An excellent review on the subject can be found in Guignard (2003). Lagrangean relaxation is a useful technique for problems with complicating constraints. To illustrate how the technique works, assume we have a problem of the following form:

$$
\min _{x}\{f x \mid A x \leq b, C x \leq d, x \in X\}
$$

where $X=\mathfrak{R}_{+}^{n-p} \times\{0,1\}^{p}$, and where the constrains $A x \leq b$ are considered to be complicating. Let $\lambda$ be a vector of nonnegative Lagrange multipliers. The Lagrangean relaxation of $(P)$ is given by the following problem:

$$
\min _{x}\{f x+\lambda(A x-b), \mid C x \leq d, x \in X\} .
$$

If we denote the feasible set of a problem as $F(\cdot)$ and the optimal value as $v(\cdot)$, then $F(P) \subseteq F\left(L R_{\lambda}\right)$ and $v(P) \geq v\left(L R_{\lambda}\right)$ for any $\lambda$. In other words, $\left(L R_{\lambda}\right)$ is a relaxation of $(P)$ and $v\left(L R_{\lambda}\right)$ is a lower bound on $v(P)$. The best bound we can obtain for $\left(L R_{\lambda}\right)$ is solving the Lagrangean dual, that is,

$$
\max _{\lambda}\left\{\min _{x}\{f x+\lambda(A x-b), \mid C x \leq d, x \in X\}\right\}=\max _{\lambda} v\left(L R_{\lambda}\right) .
$$

The following theorem by Geoffrion (1974) establishes the equivalence between $(D)$ and a primal relaxation of $(P)$. Let $C o\{\cdot\}$ denote the convex hull of $\{\cdot\}$. Then,

$$
\max _{\lambda} v\left(L R_{\lambda}\right)=D=\min _{x}\{f x \mid A x \leq b, x \in C o\{x \in X \mid C x \leq d\}\}
$$

We use this equivalence result in deriving the theoretical result explained in the next section. Non-convexities such as binary variables in an MILP problem give rise to a difference between the optimal solution of $(D)$ and $(P)$; this difference is called a duality gap. 


\section{Theoretical properties of temporal and spatial decomposition}

In this section we present a theoretical result that shows that the bound on the optimal solution of problem (1) obtained by solving the temporal Lagrangean dual is at least as tight as the bound obtained by solving the spatial dual. The qualitative explanation is that in spatial decomposition the material balances between plants and markets are relaxed, while in temporal decomposition the plant-market balances are enforced, although the inventory constraints are relaxed. Intuitively one expects that relaxing the inventory constraints has a smaller impact than relaxing the mass balance between production sites and markets.

The theoretical result relies on the assumption that a larger feasible region in the space of product sales $(s l)$ leads to a higher profit $\pi$. Therefore, the key idea is to work in the space of the sales, $s l$, and the binary variables, $s t p$, to derive the result. The first step is to show that the projection of the linear relaxation of temporal decomposition $T L_{\lambda}$ onto the $(s l, s t p)$ space is at least as small as the projection of the linear relaxation of spatial decomposition $S L_{\lambda}$. . Since stp are the only binary variables, we proceed to show that the projection of the convex hull of $T L_{\lambda}$ onto the $(s l, s t p)$ space is at least as small as the projection of the convex hull of $S L_{\lambda}$. These first two steps are illustrated in Figures 2(a) and 2(b) for a two dimensional case. Next, the convex hulls of both Lagrangean problems $\left(T L_{\lambda}\right.$ and $\left.S L_{\lambda}\right)$ are intersected with the constraints relaxed in temporal and spatial decomposition: $\operatorname{Co}\left\{T L_{\lambda}\right\} \cap v=\bar{v} \cap \operatorname{trn}=\overline{\operatorname{trn}}$, and $\operatorname{Co}\left\{S L_{\lambda}\right\} \cap f=\bar{f}$. Once more we show that the projection of $\operatorname{Co}\left\{T L_{\lambda}\right\} \cap v=\bar{v} \cap \operatorname{trn}=\overline{\operatorname{trn}}$ onto $(s l, s t p)$ is tighter than the projection of $\left.\operatorname{Co}_{S L_{\lambda}}\right\} \cap f=\bar{f}$. From the result by Geoffrion (1974), explained in the previous background section, we can conclude that the temporal Lagrangean dual projected onto $(s l, s t p)$ is at least as tight as projection of the spatial Lagrangean dual. It follows that sales in the optimal solution to the spatial dual are equal to or greater than the sales in the optimal solution to the temporal dual. Thus, the upper bound of temporal decomposition is tighter. The remainder of this section formalizes the outline of the reasoning described above. 
(a)

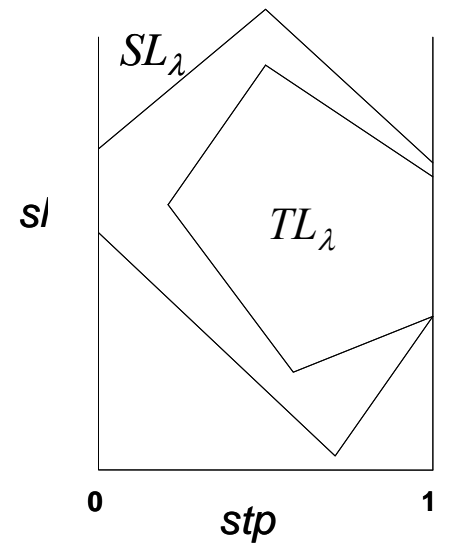

(b)

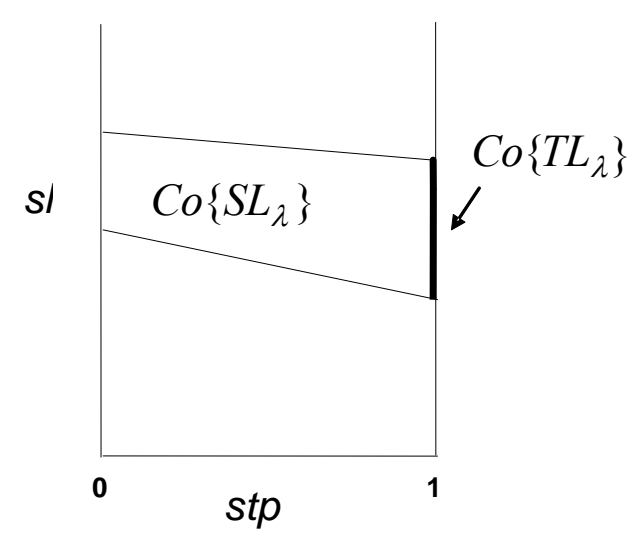

Fig. 2 Two dimensional illustration of the projections of both duals onto $(s l, s t p)$

Theorem: Temporal decomposition provides an upper bound for problem (1) that is at least as tight as that obtained by spatial decomposition. This statement is equivalent to $v\left(D^{t}\right) \leq v\left(D^{s}\right)$.

Before proving the theorem we provide some important definitions.

The temporal and spatial Lagrangean duals $\left(D^{t}\right.$ and $D^{s}$ respectively) are defined as follows.

$$
\begin{aligned}
D^{t} \equiv & \min _{\lambda v, \lambda t r} v\left(T L_{\lambda}\right)= \\
& \min _{\lambda v, \lambda t r}\left(\max _{s l, x, v, v, f, s t p, t r n, \overline{t r n}}\left\{\pi^{t}(\lambda v, \lambda t r) \text { s.t }(1.2 b),(1.3),(1.4),(1.5 b),(1.6)-(1.13)\right\}\right)
\end{aligned}
$$

and

$$
\begin{aligned}
D^{s} \equiv & \min _{\lambda f} v\left(S L_{\lambda}\right)= \\
& \min _{\lambda f}\left(\max _{s l, x, v, f, f, s t p, t r n}\left\{\pi^{s}(\lambda f) \text { s.t }(1.2)-(1.6 b),(1.7)-(1.13)\right\}\right),
\end{aligned}
$$

where $\pi^{t}$ and $\pi^{s}$ are the profit obtained in temporal decomposition $T L_{\lambda}$ and spatial decomposition $S L_{\lambda}$, respectively. 
- $F^{L P}$ is the linear programming relaxation of $F$.

- $\quad p F=\operatorname{proj}_{s l, s t p} F=\{(s l, s t p):(s l, x, v, f, s t p, t r n) \in F\}$, is the projection of $F$ onto the space of product sales and binary variables (set-ups).

Proof: By Fourier-Motzkin elimination, we can obtain the projections of the feasible regions of the linear relaxations of spatial $\left(S L_{\lambda}\right)^{L P}$ and temporal $\left(T L_{\lambda}\right)^{L P}$ decomposition onto the sales and set-up space $(s l, s t p)$. These projections onto the sales and setup variable spaces are shown below.

Projection of the linear relaxation of $F\left(S L_{\lambda}\right)$

$$
\begin{array}{lll}
b s_{t}^{s} s t p_{t}^{s} \leq L_{t} & \forall t \in T, s \in S & (p S .1) \\
C s_{t}^{s}\left(s t p_{t}^{s}\right) \leq e_{t}^{s} & \forall t \in T, s \in S & (p S .2) \\
s l_{t}^{s, m} \leq f_{s, m}^{U B} & \forall t \in T, s \in S, m \in M & (p S .3) \\
\sum_{s \in S} s l_{t}^{s, m} \leq d_{t}^{m} & \forall t \in T, m \in M & (p S .4) \\
s l_{t}^{s, m} \in \mathfrak{R}_{+}^{|l|} & & \\
0 \leq s t p_{t}^{s} \leq 1 & \forall t \in T, s \in S, m \in M & (p S .5) \\
& & (p S .6)
\end{array}
$$

Since the mass balance between plants and markets is broken, the only constraints on the set-ups are that they should not take longer than the length of the time period as shown in equation ( $p S .1)$, and that hey should correspond to a feasible sequence, as given by equation $(p S .2)$. However, there is no relationship between the products shipped to markets and the products produced in the plants. Equations $p S .3$ and $p S .4$ show that sales are only limited by transportation capacity $f^{U P}$, and by forecast, $d$.

Projection of the linear relaxation of $F\left(T L_{\lambda}\right)$

$$
b s_{t}^{s} \operatorname{stp}_{t}^{s} \leq L_{t} \quad \forall t \in T, s \in S \quad(p T .1)
$$




$$
\begin{array}{ll}
C s_{t}^{s}\left(s t p_{t}^{s}\right) \leq e_{t}^{s} & \forall t \in T, s \in S \\
s l_{t}^{s, m} \leq f_{s, m}^{U B} & \forall t \in T, s \in S, m \in M \quad(p T .3) \\
\sum_{s \in s} s l_{t}^{s, m} \leq d_{t}^{m} & \forall t \in T, m \in M \\
s l_{t}^{s, m} \in \mathfrak{R}_{+}^{|l|} & \forall t \in T, s \in S, m \in M \quad(p T .4) \\
0 \leq s t p_{t}^{s} \leq 1 & \forall t \in T, s \in S \\
\sum_{m \in M} a^{s} s l_{t}^{s, m}-\sum_{m \in M} a^{s} v_{s}^{U P}+b s_{t}^{s} s t p_{t}^{s} \leq L_{t} & t=2, \ldots, T, s \in S \\
\sum_{m \in M} a^{s} s l_{1}^{s, m}-\sum_{m \in M} a^{s} v_{0}^{s}+b s_{1}^{s} s t p_{1}^{s} \leq L_{1} & (p T .7) \\
\sum_{m \in M} s l_{t}^{s, m}-v_{s}^{U B} \leq x_{s}^{U B} s t p_{t}^{s} & \forall s \in S \\
\sum_{m \in M} s l_{1}^{s, m}-v_{s}^{U B} \leq x_{s}^{U B} s t p_{1}^{s} & t=2, \ldots, T, s \in S
\end{array}
$$

From the above equations, temporal decomposition setups take less than the length of the time period and correspond to feasible sequences; also, sales are less than or equal to the distribution capacity and forecast. This means that all equations in the projection of spatial decomposition are included in temporal decomposition as can be seen in equations $p T .1$ - pT.6. In addition, since the mass balance between plants and markets must be satisfied, equations $p T .7-p T .10$ establish relationships between setups and final product sales. These equations represent the projection of the production constraints. Note that the only impact of relaxing inventory constraints is that an amount equal to the maximum inventory holding capacity $v_{s}^{U B}$ can be sold without having to be produced, as shown in equations $p T .9$ and $p T .10$.

Since (pS.1)-(pS.6) are equivalent to $(p T .1)-(p T .6)$, and $p T$ has additional constraints, it follows that $p F\left(T L_{\lambda}\right)^{L P} \subseteq p F\left(S L_{\lambda}\right)^{L P}$. Recall that $p F\left(S L_{\lambda}\right)^{L P}$ and $p F\left(T L_{\lambda}\right)^{L P}$ are projections onto the space $(s l, s t p)$, and that the only integer variables in 
the MILP problem are the setup variables stp. Therefore, we can establish the following implication:

$p F\left(T L_{\lambda}\right)^{L P} \subseteq p F\left(S L_{\lambda}\right)^{L P} \Rightarrow p \operatorname{Co}\left\{T L_{\lambda}\right\} \subseteq p \operatorname{Co}\left\{S L_{\lambda}\right\}$.

Now, constraint (1.6) in the temporal Lagrangean relaxation, $T L_{\lambda}$, allows us to make the following statement: $(s l, f) \in F\left(T L_{\lambda}\right) \Rightarrow(s l, f) \in\{(s l, f): s l=f\}$, or equivalently, $(s l, f) \in F\left(T L_{\lambda}\right) \Rightarrow(s l, f, \bar{f}) \in\{(s l, f, \bar{f}): s l=\bar{f}, f=\bar{f}\}$.

This statement together with (4) gives:

$\left.p \operatorname{Co}\left\{T L_{\lambda}\right\} \subseteq p\left[\operatorname{Co}\left\{S L_{\lambda}\right\} \cap\{(f, \bar{f}): f=\bar{f})\right\}\right]$.

If the right-hand-side of expression (5) remains unchanged, any set obtained by restricting the left-hand-side is still a subset of the right-hand side. Thus, we can state that:

$p\left[\operatorname{Co}\left\{T L_{\lambda}\right\} \cap\{(\operatorname{trn}, \overline{\operatorname{trn}}, v, \bar{v}): \operatorname{trn}=\overline{\operatorname{trn}}, v=\bar{v}\}\right] \subseteq p\left[\operatorname{Co}\left\{S L_{\lambda}\right\} \cap\{(f, \bar{f}): f=\bar{f}\}\right]$.

According to the equivalence result of Geoffrion (1974),

$\operatorname{Co}\left\{T L_{\lambda}\right\} \cap\{(\operatorname{trn}, \overline{\operatorname{trn}}, v, \bar{v}): \operatorname{trn}=\overline{\operatorname{trn}}, v=\bar{v}\} \equiv F\left(D^{t}\right)$

and

$\operatorname{Co}\left\{S L_{\lambda}\right\} \cap\{(f, \bar{f}): f=\bar{f}\} \equiv F\left(D^{s}\right)$

From (7) and (8), we can restate (6) as follows,

$p\left[F\left(D^{t}\right)\right] \subseteq p\left[F\left(D^{s}\right)\right]$.

The relationship in (9) means that the feasible region of $D^{s}$ in the sales space is at least as large as that of $D^{t}$. Given the assumption that a larger feasible region in the space of product sales leads to higher profit, it then follows that $v\left(D^{t}\right) \leq v\left(D^{s}\right)$.

The significance of this theorem is that it establishes that the dual solution of the temporal decomposition yields tighter or at least as tight an upper bound on the profit in the planning problem in (1) as the dual solution of the spatial decomposition. 


\section{An accelerating technique for the solution of $\left(T L_{\lambda}\right)$ and $\left(S L_{\lambda}\right)$}

While the theoretical results in the first part of this paper support the use of temporal decomposition, we focus in this part in ways of selecting the Lagrange multipliers in order to accelerate the convergence in both temporal and spatial decompositions. The general idea is to use the economic interpretation of the Lagrange multipliers to limit their search space by setting upper and lower bounds on their possible values. The economic interpretation of the Lagrange multipliers, or dual variables, in linear programming (LP) is well understood (Dorfman et al., 1958; Chvátal, 1983). Williams (1996) expanded these ideas to mixed integer programming. In this work, we exploit the economic interpretation of Lagrange multipliers given in LP duality theory. We use this interpretation to set bounds on the possible values of the Lagrange multipliers of some of the constraints relaxed in temporal decomposition $\left(T L_{\lambda}\right)$ and spatial decomposition $\left(S L_{\lambda}\right)$. Since the constraints that we consider here are written only in terms of continuous variables, we propose to use these bounds even if $\left(T L_{\lambda}\right)$ and $\left(S L_{\lambda}\right)$ are MILP problems. This idea is developed and discussed in great detail in Trotter (2009. First, we review the interpretation of the Lagrange multipliers as dual variables in a linear programming problem. Then, we explain how to exploit the economic interpretation of the dual variables of the relaxed constraints to set valid bounds for the magnitude of the Lagrange multipliers. The role of the set of active constraints is explored by analyzing two limiting scenarios: sales are limited by production capacity, and sales are limited by market demand. In each scenario the set of active constraints in planning problem (1) is different, and thus, the values of the optimal Lagrange multipliers are also different.

\section{Lagrange multipliers as dual variables}

Consider the optimization problem $(P)$ :

$$
\min _{x}\{\varphi x \mid A x \leq b, C x \leq d, x \in X\} .
$$

The Lagrangean relaxation of $(P)$ is:

$$
\min _{x}\{\varphi x+\lambda(A x-b), \mid C x \leq d, x \in X\}
$$

Let $x$ be the production of a certain $\operatorname{good}$ and $\varphi$ its cost. The Lagrange multiplier $\lambda$ in $\left(L R_{\lambda}\right)$ imposes a penalty for violating constraint $A x \leq b$ in $(P)$. In duality theory, $\lambda$ is the 
dual variable associated with constraint $A x \leq b$; the optimal value of the dual variable, represents the added value if constraint $A x \leq b$ is violated by one unit, and if the optimal active set remains the same. If $\left(L R_{\lambda}\right)$ is linear and continuous, and $\lambda$ is set to the optimal value of the dual variable associated with $A x \leq b$, then the optimal value of $(P)$ and $\left(L R_{\lambda}\right)$ are the same. This last result does not hold, in general, for mixed-integer linear programming (MILP) problems. In this case the optimal value of $\left(L R_{\lambda}\right)$ is generally larger than the optimal value of $(P)$. Since the Lagrange multiplier is still a penalty for the violation of constraint $A x \leq b$. Given the structure of problem (1), we propose to use the economic interpretation of the dual variables of its linear relaxation, as a basis for setting bounds on the optimal penalty $\lambda$ in $\left(L R_{\lambda}\right)$, even if our problem is an MILP.

\section{Economic interpretation of the Lagrange multipliers}

Temporal and spatial decomposition involve the relaxation of constraints (1.14)-(1.15) and (1.16), respectively. However, we do not derive bounds on the multipliers of the sequencing constraint (1.15) due to the difficulty in finding the economic interpretation of its multipliers. Relaxing constraints (1.14) or (1.15) can be represented as breaking some of the links in the multi-period network structure of production sites and markets. Figures 3 and 4 illustrate this representation.

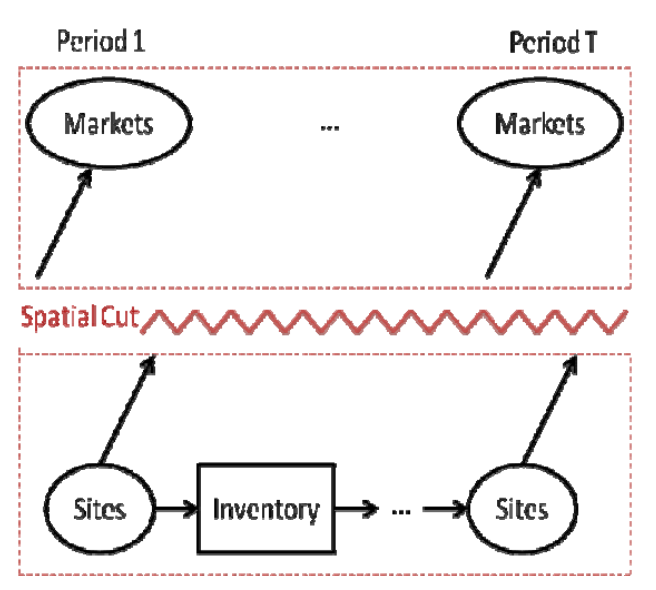

Fig. 3: Spatial decomposition

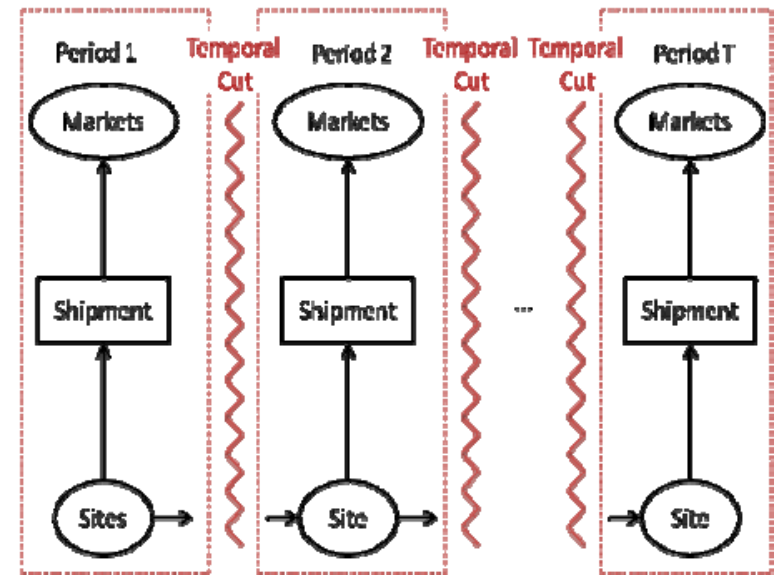

Fig. 4: Temporal decomposition 
When temporal decomposition is considered, in order to penalize the violation of constraint (1.14), the term $\sum_{t \in T} \sum_{s \in S} \lambda v_{t}^{s}\left(v_{t}^{s}-\bar{v}_{t}^{s}\right)$ is added to the objective function of $T L_{\lambda}$. The multipliers $\lambda v_{t}^{s}$ act as a selling price for $v_{t}^{s}$ or a buying price for $\bar{v}_{t}^{s}$ in a hypothetical external market. Our main idea in this section of the paper is that there is a market price $\lambda v_{t}^{s}$ that makes the profit in the decomposed system in Figure 4 very similar to the profit in the original, full space, planning problem (1). For instance, if integer variables are relaxed or eliminated from (1), a market value $\lambda v_{t}^{s}$ equal to the optimal dual variable of (1.14) would result in the same profit for temporal decomposition $T L_{\lambda}$ and for problem (1). Similarly, in the objective function of spatial decomposition $S L_{\lambda}, \lambda f_{t}^{s, m}$ represents the selling price of $f_{t}^{s, m}$ and the buying price of $\bar{f}_{t}^{s, m}$ in a hypothetical external market.

Our objective is to find the market values for $\bar{v}_{t}^{s}, v_{t}^{s}, \bar{f}_{t}^{s, m}$, and $f_{t}^{s, m}$ that make the optimal value of $\left(T L_{\lambda}\right)$ and $\left(S L_{\lambda}\right)$ as close as possible to the optimal value of problem (1). Notice that, since (1) is an MILP problem, there is a duality gap that prevents the optimal values of $T L_{\lambda}$ and $S L_{\lambda}$ to be identical to the solution of problem (1).

The set of active constraints in the optimal solution to Problem (1) results in different market prices $\lambda v_{t}^{s}$ and $\lambda f_{t}^{s, m}$ in the decomposed problems. Since, in general, it is impossible to know the optimal active set a priori, we analyze two extreme scenarios that correspond to completely different active sets, and determine the corresponding market prices $\lambda v_{t}^{s}$ and $\lambda f_{t}^{s, m}$. The two scenarios that we propose for problem (1) are the following: (a) the market demand exceeds the production capacity of the manufacturing sites for all time periods, and (b) the production capacity exceeds the market demand for every time period. The market values for these two extreme cases are used to set rigorous bounds on the multipliers as described below.

To find the upper bounds on the multipliers we use a value chain representation of the multi-site, multi-period network. For instance, assume that products in the network flow from stage 0 to stage $i$, and then from node $i$ to node $n$. The flow through each of these stages, or nodes, adds value to the products; the collection of value-adding stages in 
the network represents the value chain of the goods produced, distributed, and sold in the multi-site, multi-period network as shown in Figure 5.

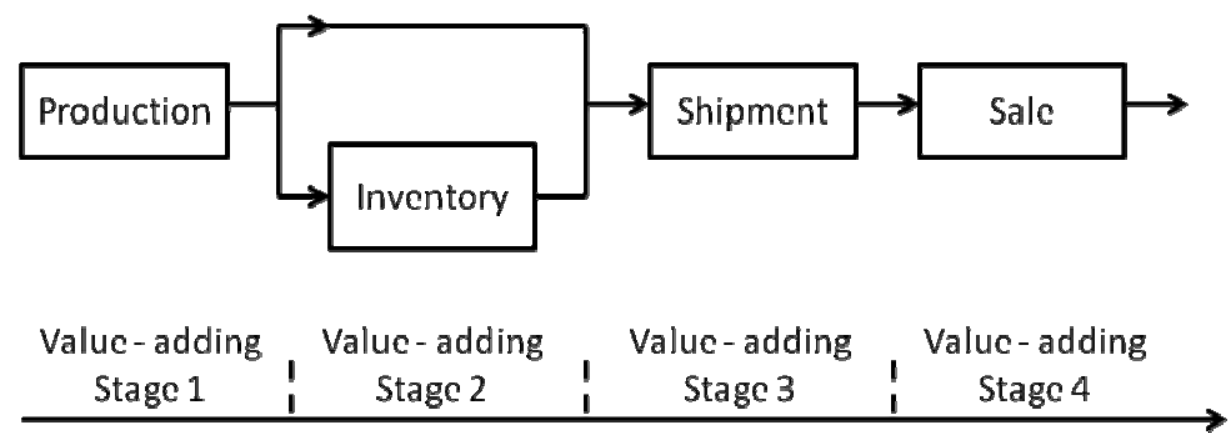

Fig.5: Value-adding chain

Stage $i$ in the network takes a product with a price equal to the value added up to stage $i-1$, and transfers the product to stage $i+1$ with a price equal to the price at which $i$ took the product, plus the value it adds in the value chain. The value of a good exchanged between $i$ and $i+1$ is commonly referred to as its transfer price. Figures 3 and 4 show how decomposition strategies can be represented as breaking a material flow in the network. Ideally, the optimal Lagrange multipliers $\lambda f_{t}^{s, m}$ and $\lambda v_{t}^{s}$ in $\left(S L_{\lambda}\right)$ and in $\left(T L_{\lambda}\right)$ should be equal to the transfer price at the links broken in Figures 3 and 4, respectively.

There are two ways of analyzing the transfer prices in the value chain formed by the multi-site, multi-period network. We can set the value of the raw material for the production sites to zero (this is not the "real" price, but our baseline for the analysis) so that every step after that adds value to the product. To illustrate this idea, consider a small model with two time periods, one site and one market. Figure 6 present the analysis of the transfer prices starting with the production sites. In this figure, $\alpha^{\prime}$ denotes the costs that arise within a site that include the manufacturing cost $\alpha$ and the cost of transitions divided by the total production, and hence, it corresponds to the value added per unit of product at the production site. This analysis is called forward analysis and it is illustrated in Figure 6. Figure 7 shows the transfer prices obtained by using an alternative we call the backwards analysis. The idea is that products have a maximum value at the markets, and the transfer price at each link in the value chain is determined by going backwards. At the 
shipment stage, the value added is equal to the unit shipment cost $\gamma$. The same concepts can be extended to a multi-period network by understanding that a unit of product is transferred to the next period by going through an inventory stage.

In the case of scenario (a) where the production capacity limits the sales of finished product in all time periods, the sites limit the production and the markets become the initial stage with respect to the product value. Therefore, in this case a backwards approach has to be used to determine the product value at any given stage in the supply chain. In scenario (b), where the sales are limited by the market demand, the markets become the limiting stages for production in the supply chain. Therefore, the production sites must be the initial stages, which make it necessary to set the product values with the forward approach.

In spatial decomposition the link between the production sites and the shipment node in the value chain is broken. Consequently, the product value of interest for setting the bounds on the Lagrange multipliers is $\alpha$, following the forward approach in Figure 6, or $\beta-\gamma$, following the backward approach in Figure 7 , depending on whether market demand or production capacity limits the total sales. In temporal decomposition the link between production sites and inventory nodes in the value chain is broken. As a result, the temporal sub-problems are set up such that, again, the product value of interest is $\alpha$ ' or $\beta-\gamma$. Figures 3 and 4 illustrate the exact way the links are broken in spatial and temporal decomposition, respectively. When comparing these last two figures with Figures 6 and 7, it becomes apparent that the transfer prices have the same value for both spatial and temporal decomposition. Therefore, the transfer prices do not depend on the decomposition scheme chosen.

Finally, regardless of the active set of constraints, or the choice of decomposition scheme, a rigorous lower bound on the Lagrange multipliers of constraints (1.14) and (1.16) can be set to zero, since the market prices of the goods produced and distributed should be nonnegative. 


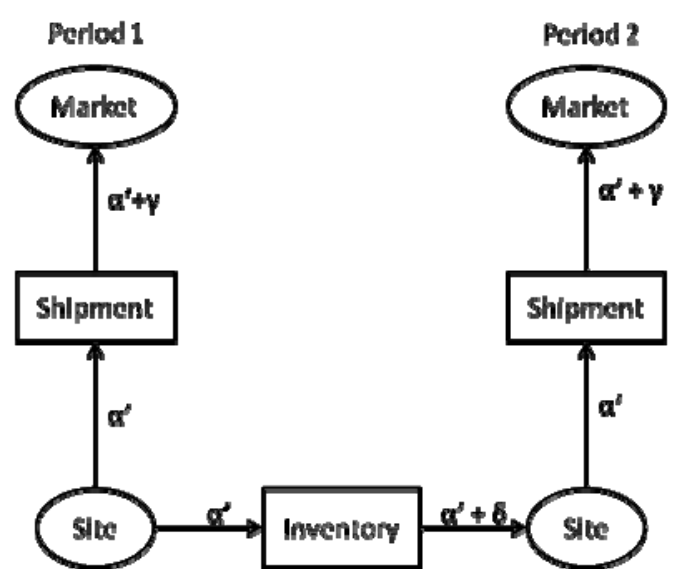

Fig. 6: Product values for forward analysis

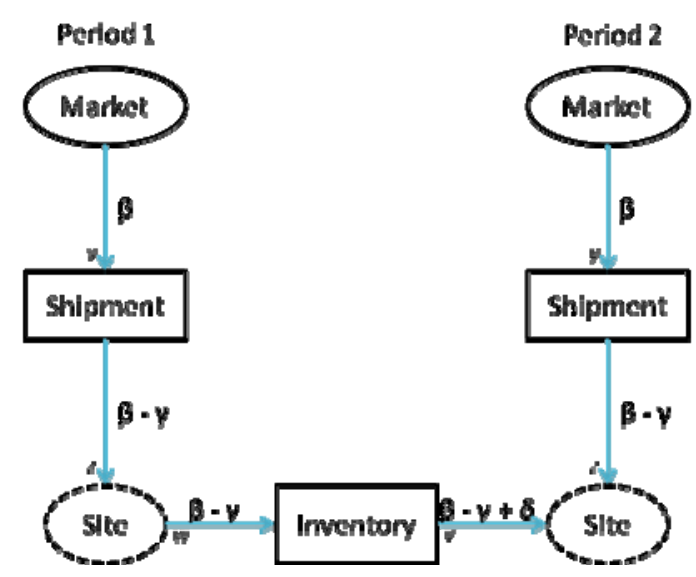

Fig. 7: Product values for backward analysis

\section{Algorithm to set valid bounds on the Lagrange multipliers}

In this section we generalize the algorithm for any problem size, and we derive valid bounds for any set of active constraints.

Step 1: Obtaining the transfer prices for the scenario of limiting production capacity

In a general network, there are several production sites serving different markets, and producing a variety of products. Therefore, the upper bound on the transfer prices obtained as $\beta-\gamma$ in the small example of the previous section is generalized as follows:

$$
\max _{m, i, t}\left\{\beta_{t}^{m, i}\right\}-\min _{m, s, i, t}\left\{\gamma_{t}^{m, s, i}\right\} \text {. }
$$

Step 2: Obtaining the transfer prices for the scenario of limiting market demand

In previous sections we found that in this limiting scenario, the transfer price is equal to a manufacturing unit cost that includes transition costs $\left(\alpha^{\prime}\right)$. To calculate the contribution of the transitions, we distribute the transitions costs incurred during the time period among all the products produced. In order to do this, we need to know the amount of products produced and the maximum possible transition cost that have to be paid. In the case were production is fully limited by demand, the total amount of products produced is $\sum_{t=1}^{|T|} \sum_{i=1}^{|I|} \sum_{m=1}^{|M|} d_{t, i}^{m}$. Assuming that for every site and product in all time periods, the maximum 
transition cost is $\max _{s, i, i^{\prime}, t}\left\{T C_{t}^{s, i, i^{\prime}}\right\}$, the upper bound on transition costs is $\left.\max _{s, i, i^{\prime}, t}\left\{T C_{t}^{s, i, i^{\prime}}\right\}|| T \mid-1\right)|S||I|$. Then, distributing the transition costs among all manufactured product is equal to $\frac{\max _{s, i, i^{\prime}, t}\left\{T C_{t}^{s, i, i^{\prime}}\right\}(|T|-1)|S||I|}{\sum_{t=1}^{|T|} \sum_{i=1}^{|I|} \sum_{m=1}^{|M|} d_{t, i}^{m}}$, and the cost $\alpha^{\prime}$ is calculated as follows:

$$
\alpha^{\prime}=\max _{s, i, i^{\prime}, t}\left\{\alpha_{s, i}^{t}\right\}+\frac{\max _{s, i, i^{\prime}, t}\left\{T C_{t}^{s, i, i^{\prime}}\right\}(|T|-1)|S||I|}{\sum_{t=1}^{|T|} \sum_{i=1}^{|I|} \sum_{m=1}^{|M|} d_{t, i}^{m}} .
$$

In the equation above, the same cost, $\alpha$, is used in the calculation of the market value, $\alpha$, for all products. However, as every site may have different shipment costs $\gamma$ to the same markets $m$, the value of the product has to be adjusted by the respective difference in shipment costs between the basis site with cost $\alpha^{\prime}$ and the other sites. To cover all scenarios, we set this correction term $\Delta \gamma$ to its maximum, i.e. $\Delta \gamma=\gamma_{\max }-\gamma_{\min }$. Therefore, the upper bound on the transfer prices can be set to

$$
\alpha^{\prime}+\max _{m, s, i, t}\left\{\gamma_{t}^{m, s, i}\right\}-\min _{m, s, i, t}\left\{\gamma_{t}^{m, s, i}\right\} \text {. }
$$

Step 3: Adjusting for the intermediate case - some periods limited by capacity, others by market demand.

When some time periods are limited by market demand and some others by production capacity, there is an incentive for carrying inventory from the periods where sales are limited by market demand to the periods where sales are limited by production capacity. The transfer of a product from one time period to the next as inventory, increases its value in the value chain by an amount equal to $\delta$. Since we do not know for how many time periods we will have to carry a product in inventory, we assume the worst case where a product is carried in inventory from the first time period to the last time period. In this case the value of the product in the value chain is increased by a magnitude equal to $\delta(|T|-1)$. We are interested in finding valid upper bounds for the transfer prices, so we choose the largest inventory holding cost in the network: $\max _{s, i, t}\left\{\delta_{t}^{s, i}\right\}\{|T|-1)$. Finally this 
term is added to the formula for obtaining an upper bound on the transfer price in (a) the limiting production capacity case, and (b) the limiting market demand case.

$$
\begin{aligned}
U P_{\text {capacity }}^{\lim }= & \left.\max _{m, i, t}\left\{\beta_{t}^{m, i}\right\}-\min _{m, s, i, t}\left\{\gamma_{t}^{m, s, i}\right\}+\max _{s, i, t}\left\{\delta_{t}^{s, i}\right\}|T|-1\right) \\
U P_{\text {demand }}^{\lim }= & \max _{s, i, t}\left\{\alpha_{s, i}^{t}\right\}+\frac{\left.\max _{s, i, i^{\prime}, t}\left\{T C_{t}^{s, i, i^{\prime}}\right\}|T|-1\right)|S||I|}{\sum_{t=1}^{|T|} \sum_{i=1}^{|I|} \sum_{m=1}^{|M|} d_{t, i}^{m}} \\
& \left.+\max _{m, s, i, t}\left\{\gamma_{t}^{m, s, i}\right\}-\min _{m, s, i, t}\left\{\gamma_{t}^{m, s, i}\right\}+\max _{s, i, t}\left\{\delta_{t}^{s, i}\right\}|T|-1\right)
\end{aligned}
$$

\section{Step 4: Solving the linear relaxation of Problem (1)}

It is impossible to know the complete active set of planning problem (1) before solving it. However, it is possible to infer from the linear relaxation some useful information. The change from fractional to integral variables in (1.4) either reduces or does not change the amount of products that can be produced. Therefore, if the production time constraint (1.4) is active for all sites in the linear relaxation of the model, production capacity is also limiting in the MILP problem. In this case, the upper bound on the Lagrange multipliers corresponds to the extreme scenario of limiting production capacity. If (1.4) is not active for at least one site in the linear relaxation, we have to derive valid upper and lower bounds using transfer prices from both limiting scenarios (limiting capacity and limiting market demand). A constraint is active if its Lagrange multiplier is different from 0 . After solving the LP relaxation of Problem (1) we keep record of the marginal value (Lagrange multiplier) of constraint (1.4), for a certain time period $t$ and site $s$, by using a parameter $\mu_{t, s}$

\section{Step5: Assign bounds to the Lagrange multipliers}

First we check if constraint (1.4) is active in all time periods in the LP relaxation (i.e its multipliers $\mu_{t, s}$ is nonzero). Then we assign the upper bound on the transfer price, and thus, on the Lagrange multipliers, using the largest bound available from the limiting cases. 
If $\mu_{t, s} \neq 0 \forall t \in T$

$$
0 \leq \lambda f_{t}^{s, m}, \lambda v_{t}^{s} \leq U P_{\text {capacity }}^{\lim }
$$

Else

$$
0 \leq \lambda f_{t}^{s, m}, \lambda v_{t}^{s} \leq \max \left\{U P_{\text {capacity }}^{\lim }, U P_{\text {demand }}^{\lim }\right\}
$$

\section{Numerical Results}

Section 7 addresses four examples that use this algorithm to set bounds on the multipliers as part of the numerical solution of the Lagrangean dual in temporal and spatial decomposition. We apply the Lagrangean decomposition using the algorithmic steps described in Figure 8.

\section{Example 1}

The first example is a small and simplified version of the production planning model introduced in section 2. It involves production set-ups but no sequence-dependent changeovers. Therefore, the setup variables $s t p_{t}^{s}$ replace $t r n_{t}^{s}$ in the objective function. Appendix $\mathrm{C}$ contains the equations of this model. Example 1 consists of three time periods, three production sites, three markets and three products. It has 27 discrete variables, 316 continuous variables, and 253 constraints. The optimal solution of this problem has an objective function of $\$ 41,576$ over a 3-month-period, and the corresponding optimal production plan is shown in Figure 9. We can see in this production plan that the production capacity is fully used only in time periods 1 and 2 , while the three sites are totally or partially shut down in time period 3 . This corresponds to the mixed case where in some time periods the sales are limited (constrained) by production capacity, while in the rest sales are limited by the market demand. 


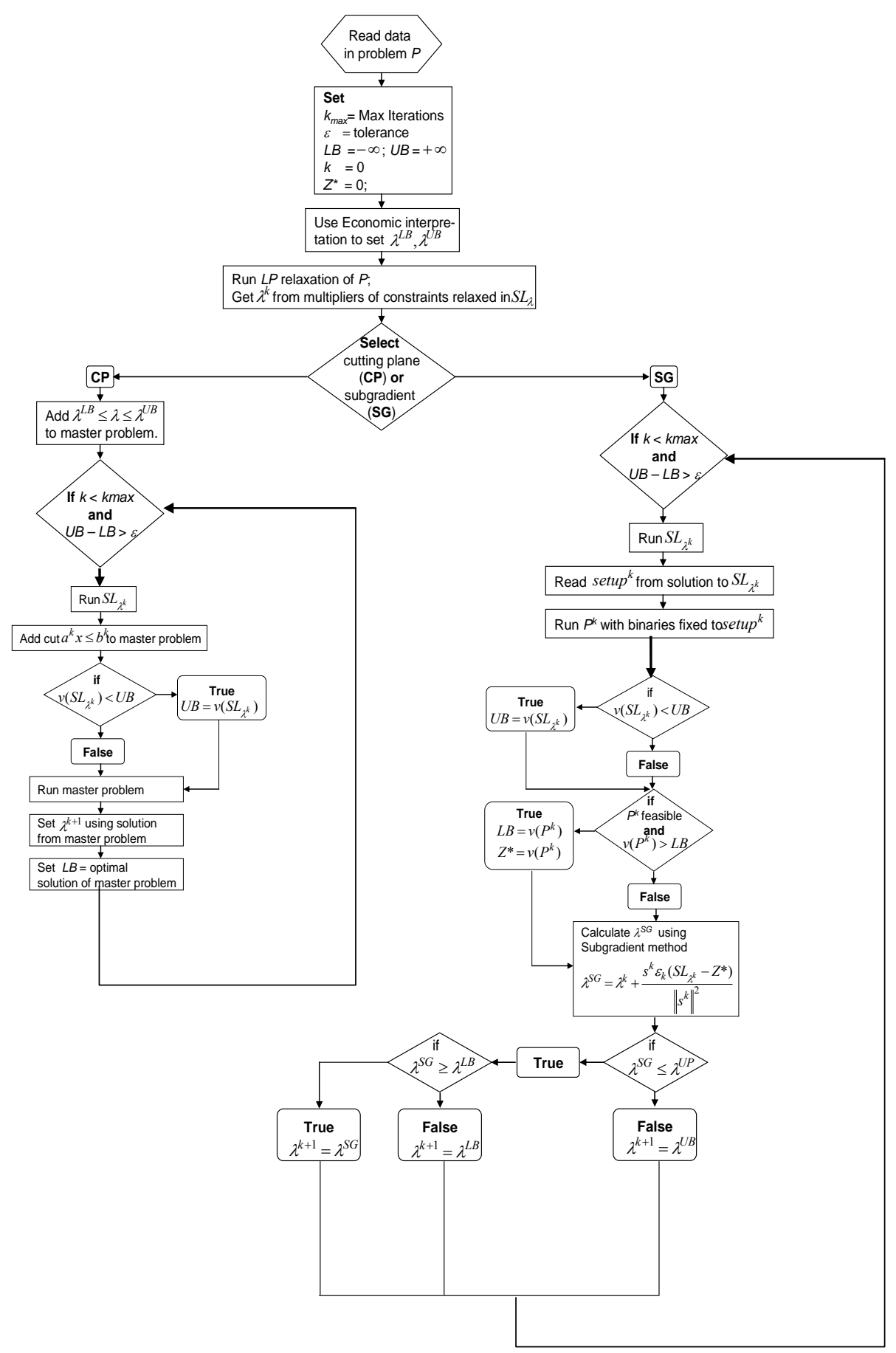

Fig. 8 Algorithm illustrated with spatial decomposition $S L_{\lambda}$

The objective of this first example is to provide numerical evidence that temporal decomposition gives tighter bounds on the full space optimal solution than spatial decomposition and that the solution of the Lagrangean dual can be sped up by using the economic bounds. Also, this example illustrates that both properties also hold true for simplified versions of problem (1). 
The Lagrangean dual in this example is solved with a cutting plane algorithm (Guignard, 2003) as described in Appendix B. This method gives a lower and an upper bound on the optimal dual solution per iteration. The convergence criterion is set to $1 \%$ gap between the two bounds. This algorithm is known to be slow to converge but guarantees finding an optimal solution to the Lagrangean dual. As we want to compare the optimal solutions for both decomposition approaches, we chose to solve the first two examples with this algorithm.

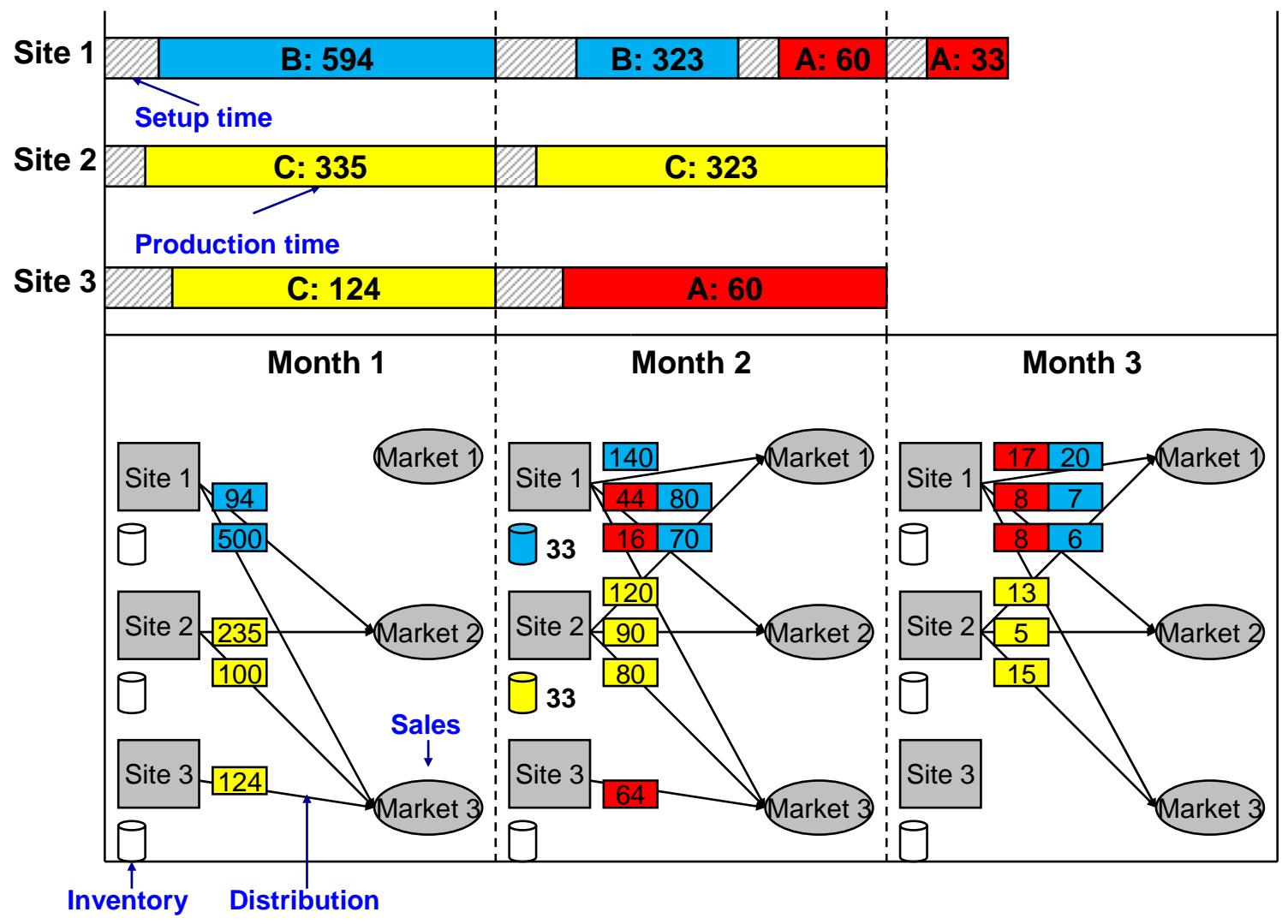

Fig. 9 Optimal production plan for Example 1

Before the problem is solved, the economic bounds are calculated in detail. Table 1a and $1 \mathrm{~b}$ list all the parameters of the model at hand. 
Table 1a: Data for Example One

\begin{tabular}{|c|c|c|c|c|c|c|c|c|c|}
\hline $\begin{array}{l}\text { Demand } \\
\text { Forecast }\end{array}$ & $\begin{array}{l}\mathrm{t}=1 \\
{[\text { ton] }}\end{array}$ & $\begin{array}{l}\mathrm{t}=2 \\
{[\text { ton] }}\end{array}$ & $\begin{array}{l}\mathrm{t}=3 \\
\text { [ton] }\end{array}$ & $\begin{array}{c}\text { Prod. } \\
\text { Rate }\end{array}$ & [ton $/ \mathrm{hr}]$ & $\begin{array}{l}\text { Setup } \\
\text { Times } \\
\end{array}$ & [hr] & $\begin{array}{c}\text { Horizon } \\
\text { Time }\end{array}$ & [hr] \\
\hline$d_{M 1, I 1}$ & 70 & 120 & 17 & $a_{S 1, I 1}$ & 0.3 & $b s_{S I, I 1}$ & 100 & $L_{t}$ & 720 \\
\hline$d_{M 2, I I}$ & 120 & 90 & 8 & $a_{S 2, I 1}$ & 0.1 & $b s_{S 2, I I}$ & 70 & & \\
\hline$d_{M 3, I I}$ & 50 & 80 & 8 & $a_{S 3, I 1}$ & 0.1 & $b s_{S 3, I I}$ & 80 & & \\
\hline$d_{M 1, I 2}$ & 90 & 140 & 20 & $a_{S 1, I 2}$ & 0.9 & $b s_{S 1, I 2}$ & 60 & & \\
\hline$d_{M 2, I 2}$ & 100 & 80 & 7 & $a_{S 2, I 2}$ & 0.5 & $b s_{S 2, I 2}$ & 100 & & \\
\hline$d_{M 3, I 2}$ & 500 & 70 & 6 & $a_{S 3, I 2}$ & 0.1 & $b s_{S 3, I 2}$ & 40 & & \\
\hline$d_{M 1, I 3}$ & 170 & 120 & 13 & $a_{S 1, I 3}$ & 0.3 & $b s_{S 1, I 3}$ & 80 & & \\
\hline$d_{M 2, I 3}$ & 600 & 90 & 5 & $a_{S 2, I 3}$ & 0.5 & $b s_{S 2, I 3}$ & 50 & & \\
\hline$d_{M 3, I 3}$ & 100 & 80 & 15 & $a_{S 3, I 3}$ & 0.2 & $b s_{S 3, I 3}$ & 100 & & \\
\hline
\end{tabular}

Table 1b: Data for Example One

\begin{tabular}{|c|c|c|c|c|c|c|c|c|c|c|c|}
\hline $\begin{array}{c}\text { Shipm. } \\
\text { Costs }\end{array}$ & $\begin{array}{c}I 1 \\
{[\$ /} \\
\text { ton] }\end{array}$ & $\begin{array}{c}I 2 \\
{[\$ /} \\
\text { ton] }\end{array}$ & $\begin{array}{c}I 3 \\
{[\$ /} \\
\text { ton] }\end{array}$ & $\begin{array}{l}\text { Prod. } \\
\text { Costs }\end{array}$ & $\begin{array}{l}{[\$ /} \\
\text { ton] }\end{array}$ & $\begin{array}{c}\text { Invent. } \\
\text { Costs }\end{array}$ & $\begin{array}{l}{[\$ /} \\
\text { ton] }\end{array}$ & $\begin{array}{c}\text { Sales } \\
\text { Prices }\end{array}$ & $\begin{array}{l}{[\$ /} \\
\text { ton] }\end{array}$ & $\begin{array}{l}\text { Setup } \\
\text { Costs }\end{array}$ & {$[\$]$} \\
\hline$\gamma_{M 1, S 1}$ & 3 & 3 & 3 & $\alpha_{S 1, I 1}$ & 5.5 & $\delta_{S 1, I I}$ & 0.1 & $\beta_{M 1, I 1}$ & 20 & $T C_{S I, I I}$ & 100 \\
\hline$\gamma_{M 1, S 2}$ & 4 & 4 & 2 & $\alpha_{S 2, I I}$ & 3.5 & $\delta_{S 2, I I}$ & 0.2 & $\beta_{M 2, I I}$ & 25 & $T C_{S 2, I I}$ & 400 \\
\hline$\gamma_{M 1, S 3}$ & 1 & 1 & 3 & $\alpha_{S 3, I I}$ & 4.5 & $\delta_{S 3, I I}$ & 0.05 & $\beta_{M 3, I I}$ & 30 & $T C_{S 3, I I}$ & 700 \\
\hline$\gamma_{M 2, S I}$ & 2 & 2 & 2 & $\alpha_{S 1, I 2}$ & 2.5 & $\delta_{S 1, I 2}$ & 0.15 & $\beta_{M 1, I 2}$ & 23 & $T C_{S 1, I 2}$ & 200 \\
\hline$\gamma_{M 2, S 2}$ & 4 & 1 & 4 & $\alpha_{S 2, I 2}$ & 4.5 & $\delta_{S 2, I 2}$ & 0.2 & $\beta_{M 2, I 2}$ & 28 & $T C_{S 2, I 2}$ & 300 \\
\hline$\gamma_{M 2, S 3}$ & 5 & 3 & 2 & $\alpha_{S 3, I 2}$ & 5.5 & $\delta_{S 3, I 2}$ & 0.15 & $\beta_{M 3, I 2}$ & 33 & $T C_{S 3, I 2}$ & 500 \\
\hline$\gamma_{M 3, S I}$ & 5 & 5 & 5 & $\alpha_{S 1, I 3}$ & 2.5 & $\delta_{S 1, I 3}$ & 0.1 & $\beta_{M 1, I 3}$ & 26 & $T C_{S 1, I 3 \$}$ & 100 \\
\hline$\gamma_{M 3, S 2}$ & 2 & 3 & 2 & $\alpha_{S 2, I 3}$ & 5.5 & $\delta_{S 2, I 3}$ & 0.25 & $\beta_{M 2, I 3}$ & 32 & $T C_{S 2, I 3}$ & 200 \\
\hline$\gamma_{M 3, S 3}$ & 1 & 1 & 5 & $\alpha_{S 3, I 3}$ & 4.5 & $\delta_{S 3, I 3}$ & 0.1 & $\beta_{M 3, I 3}$ & 37 & $T C_{S 3, I 3}$ & 400 \\
\hline
\end{tabular}

To set the bounds on the Lagrange multipliers, we can calculate from equations (11) and (12):

$$
\begin{aligned}
& U P_{\text {capacity }}^{\lim }=\max _{m, i, y}\left\{\beta_{t}^{m, i}\right\}-\min _{m, s, i, t}\left\{\gamma_{t}^{m, i}\right\}+\max _{s, i, t}\left\{\delta_{t}^{s, i}\right\} \mid(|T|-1) \\
& =37-1+0.25 \cdot(3-1)=36.5 \\
& U P_{\text {demand }}^{\lim }=U P_{\text {demand }}^{\lim }=\max _{s, i, t}\left\{\left\langle\alpha_{s, i}^{t}\right\}+\frac{\max _{s, i, i^{\prime} t}\left\{T C_{t}^{s, i, i^{\prime}}\right\}|(|T|-1) S||I|}{\sum_{t=1}^{|T|} \sum_{i=1}^{|I|} \sum_{m=1}^{|M|} d_{t, i}^{m}}\right. \\
& +\max _{m s, i, t}\left\{\gamma_{t}^{m, s, i}\right\}-\min _{m, s, i, t}\left\{\gamma_{t}^{m, s, i}\right\}+\max _{s, i, t}\left\{\delta_{t}^{s, i}\right\} \mid(T \mid-1) \\
& =5.5+\max \{63.363 ; 13.003 ; 6.826\}+5-1+0.25 \cdot(3-1)=73.636
\end{aligned}
$$


The solution of the LP relaxation of the model shows that time capacity constraint (1.4) is active for all sites in periods one and two, while it is not active in the last period. Therefore, we can set the bounds to,

$0 \leq \lambda f_{t=1}^{s, m}, \lambda f_{t=2}^{s, m}, \lambda v_{t=1}^{s}, \lambda v_{t=2}^{s} \leq U P_{\text {capacity }}^{\lim }=36.5$

$0 \leq \lambda f_{t=33}^{s, m}, \lambda v_{t=3}^{s} \leq U P_{\text {capacity }}^{\lim }=73.636$

With these bounds, both of the decomposition models can be solved. We solve the Lagrangean dual with the cutting plane algorithm, and we set the convergence criterion to less than or equal to 1\% gap between the algorithm's upper and lower bounds. Table 2a and $2 \mathrm{~b}$ summarize the performance of both decomposition schemes. Note that in Table $2 \mathrm{a}$ the bounds are not reported at their last iteration since arbitrary increments in the number of iterations are used. In both tables the economically set bounds are compared to a case where the multipliers are generically bounded between zero and $10^{6}$.

Table 2a Progress of cutting plane algorithm with and without bounded multipliers in Example 1

\begin{tabular}{|c|c|c|c|c|c|c|c|c|}
\hline & \multicolumn{4}{|c|}{ Spatial Decomposition } & \multicolumn{3}{c|}{ Temporal Decomposition } \\
\hline Iteration & Generic Bounds & \multicolumn{2}{|c|}{ Economic Bounds } & \multicolumn{2}{c|}{ Generic Bounds } & \multicolumn{2}{c|}{ Economic Bounds } \\
\hline & UB [\$] & gap [\%] & UB [\$] & gap [\%] & UB [\$] & gap [\%] & UB [\$] & gap [\%] \\
\hline $\mathbf{1}$ & 42676 & $>>100$ & 42676 & $>>100$ & 41823 & $>>100$ & 41823 & 22.1 \\
\hline $\mathbf{5 0}$ & 42676 & $>>100$ & 42676 & 17.2 & 41823 & 14 & 41786 & 2.7 \\
\hline $\mathbf{1 0 0}$ & 42676 & 55.8 & 42676 & 11.4 & 41823 & 9.3 & - & - \\
\hline $\mathbf{2 0 0}$ & 42676 & 30.6 & 42676 & 8.0 & 41708 & 2.1 & - & - \\
\hline $\mathbf{5 0 0}$ & 42676 & 11.2 & 42239 & 3.6 & - & - & - & - \\
\hline $\mathbf{1 0 0 0}$ & 42676 & 7.3 & 41767 & 1.4 & - & - & - & - \\
\hline$\sim 9500$ & 41713 & 1 & - & - & - & - & - & - \\
\hline
\end{tabular}


Table 2b Convergence of cutting plane algorithm with bounded multipliers in Example 1

\begin{tabular}{|c|c|c|}
\hline & Spatial Decomposition & Temporal Decomposition \\
\hline Iterations Generic Bounds & 9497 & 269 \\
\hline Iterations Economic Bounds & 1538 & 85 \\
\hline Upper bound on profit & $\$ 41,682$ & $\$ 41,640$ \\
\hline
\end{tabular}

In this example, both the spatial and the temporal decomposition provide tight upper bounds on the optimal solution of the full space problem, being only $0.25 \%$ and $0.15 \%$ greater than the optimal solution of this example (\$41,576/3-month-period).

Tables $2 \mathrm{a}$ and $2 \mathrm{~b}$ allow for three important observations. First, in both decompositions, initializing the multipliers with the marginal values of the related constraint is proven to be a very good starting point for the upper bound of the algorithm. Second, temporal decomposition requires significantly less cutting plane iterations for convergence; over $90 \%$ computational time can be saved. Third, the economic bounds further reduce the number of iterations by $63 \%$ for temporal and by over $82 \%$ for spatial decomposition

\section{Example 2}

The second example is a small version of a production planning model that includes sequence-dependent changeovers using the sequencing constraints included in the model shown in Appendix A. The example consists of four time periods, three production sites, two markets and three products, which results in 36 discrete variables, 793 continuous variables, and 856 constraints. The optimal solution of this problem has an objective function value of $\$ 66,810$. Again, this problem is solved using a cutting plane algorithm. Table $3 \mathrm{a}$ and $3 \mathrm{~b}$ list all the parameters for the model.

Table 3a: Data for Example Two

\begin{tabular}{|c|c|c|c|c|c|c|c|c|c|c|}
\hline $\begin{array}{l}\text { Demand } \\
\text { Forecast }\end{array}$ & $\begin{array}{l}\mathrm{t}=1 \\
\text { [ton] }\end{array}$ & $\begin{array}{l}\mathrm{t}=2 \\
{[\text { ton] }}\end{array}$ & $\begin{array}{l}\mathrm{t}=3 \\
\text { [ton] }\end{array}$ & $\begin{array}{l}\mathrm{t}=4 \\
{[\text { ton] }}\end{array}$ & $\begin{array}{c}\text { Prod. } \\
\text { Rate }\end{array}$ & $\begin{array}{l}{[\text { ton/ }} \\
\text { month] }\end{array}$ & $\begin{array}{l}\text { Setup/ } \\
\text { Trans. }\end{array}$ & [month] & $\begin{array}{c}\text { Horizon } \\
\text { Time }\end{array}$ & $\begin{array}{c}\mathrm{V}^{\mathrm{t}} \\
{[\mathrm{month}]}\end{array}$ \\
\hline$d_{M 1, I 1}$ & 350 & 350 & 300 & 150 & $a_{S 1, I 1}$ & 80 & $b s, t_{I I, I I}$ & 0 & \multirow[t]{9}{*}{$L_{t}$} & \multirow[t]{9}{*}{1} \\
\hline$d_{M 2, I 1}$ & 115 & 100 & 100 & 115 & $a_{S 2, I 1}$ & 350 & $b s, t_{I 2, I I}$ & 0.05 & & \\
\hline$d_{M 1, I 2}$ & 200 & 100 & 50 & 200 & $a_{S 3, I I}$ & 200 & $b s, t_{I 3, I I}$ & 0.05 & & \\
\hline$d_{M 2, I 2}$ & 400 & 450 & 570 & 400 & $a_{S 1, I 2}$ & 300 & $b s, t_{I 1, I 2}$ & 0.05 & & \\
\hline$d_{M 1, I 3}$ & 150 & 200 & 350 & 300 & $a_{S 2, I 2}$ & 250 & $t s, t_{I 2, I 2}$ & 0 & & \\
\hline$d_{M 2, I 3}$ & 200 & 300 & 350 & 320 & $a_{S 3, I 2}$ & 350 & $b s, t_{I 3, I 2}$ & 0.05 & & \\
\hline & & & & & $a_{S 1, I 3}$ & 300 & $b s, t_{I 1, I 3}$ & 0.1 & & \\
\hline & & & & & $a_{S 2, I 3}$ & 300 & $b s, t_{I 2, I 3}$ & 0.1 & & \\
\hline & & & & & $a_{S 3, I 3}$ & 100 & $b s, t_{I 3, I 3}$ & 0 & & \\
\hline
\end{tabular}


Table 3b: Data for Example Two

\begin{tabular}{|c|c|c|c|c|c|c|c|c|c|c|c|}
\hline $\begin{array}{c}\text { Shipm. } \\
\text { Costs }\end{array}$ & $\begin{array}{c}\forall \mathrm{t}, \\
I 1 \\
{[\$ /} \\
\text { ton] }\end{array}$ & $\begin{array}{c}\forall \mathrm{t}, \\
I 2 \\
{[\$ /} \\
\text { ton] }\end{array}$ & $\begin{array}{l}\forall / t \\
I 3 \\
{[\$ /} \\
\text { ton] }\end{array}$ & $\begin{array}{l}\text { Prod. } \\
\text { Costs }\end{array}$ & $\begin{array}{l}{[\$ /} \\
\text { ton] }\end{array}$ & $\begin{array}{c}\text { Invent. } \\
\text { Costs }\end{array}$ & $\begin{array}{l}{[\$ /} \\
\text { ton] }\end{array}$ & $\begin{array}{l}\text { Sales } \\
\text { Prices }\end{array}$ & $\begin{array}{l}{[\$ /} \\
\text { ton] }\end{array}$ & $\begin{array}{l}\text { Setup } \\
\text { Costs }\end{array}$ & {$[\$]$} \\
\hline$\gamma_{M 1, S 1}$ & 4 & 2 & 5 & $\alpha_{S I, I 1}$ & 6.5 & $\delta_{S 1, I I}$ & 0.1 & $\beta_{M 1, I I}$ & 20 & $T C_{I I, I I}$ & 0 \\
\hline$\gamma_{M 2, S I}$ & 5 & 5 & 2.5 & $\alpha_{S 2, I I}$ & 6 & $\delta_{S 2, I I}$ & 0.05 & $\beta_{M 2, I 1}$ & 18 & $T C_{I 2, I I}$ & 150 \\
\hline$\gamma_{M 1, S 2}$ & 3 & 1 & 4 & $\alpha_{S 3, I l}$ & 3 & $\delta_{S 3, I I}$ & 0.1 & $\beta_{M 1, I 2}$ & 22.5 & $T C_{I 3, I I}$ & 100 \\
\hline$\gamma_{M 2, S 2}$ & 6.5 & 6 & 2 & $\alpha_{S 1, I 2}$ & 5 & $\delta_{S 1, I 2}$ & 0.1 & $\beta_{M 2, I 2}$ & 22 & $T C_{I I, I 2}$ & 100 \\
\hline$\gamma_{M 1, S 3}$ & 3 & 2 & 6.5 & $\alpha_{S 2, I 2}$ & 5 & $\delta_{S 2, I 2}$ & 0.2 & $\beta_{M 1, I 3}$ & 28 & $T C_{I 2, I 2}$ & 0 \\
\hline$\gamma_{M 2, S 3}$ & 4 & 5.5 & 3 & $\alpha_{S 3, I 2}$ & 3 & $\delta_{S 3, I 2}$ & 0.2 & $\beta_{M 2, I 3}$ & 30 & $T C_{I 3, I 2}$ & 250 \\
\hline & & & & $\alpha_{S 1, I 3}$ & 5.5 & $\delta_{S 1, I 3}$ & 0.05 & & & $T C_{I I, I 3}$ & 200 \\
\hline & & & & $\alpha_{S 2, I 3}$ & 3.5 & $\delta_{S 2, I 3}$ & 0.1 & & & $T C_{I 2, I 3}$ & 300 \\
\hline & & & & $\alpha_{S 3, I 3}$ & 4.5 & $\delta_{S 3, I 3}$ & 0.05 & & & $T C_{I 3, I 3}$ & 0 \\
\hline
\end{tabular}

With this, we can calculate from equations (11) and (12):

$$
\begin{aligned}
& U P_{\text {capacity }}^{\lim }=\max _{m, i, t}\left\{\beta_{t}^{m, i}\right\}-\min _{m, s, i, t}\left\{\gamma_{t}^{m, i}\right\}+\max _{s, i, t}\left\{\delta_{t}^{s, i}\right\}(|T|-1) \\
& =30-1+0.2 \cdot(4-1)=29.6 \\
& U P_{\text {demand }}^{\lim _{s, i, t}}=\max _{s, i}\left\{\alpha_{s, i}^{t}\right\}+\frac{\left.\max _{s, i, i^{\prime}, t}\left\{T C_{t}^{s, i, i^{\prime}}\right\}|| T \mid-1\right)|S||I|}{\sum_{t=1}^{|T|} \sum_{i=1}^{|I|} \sum_{m=1}^{|M|} d_{t, i}^{m}} \\
& +\max _{m, s, i, t}\left\{\gamma_{t}^{m, s, i}\right\}-\min _{m, s, i, t}\left\{\gamma_{t}^{m, s, i}\right\}+\max _{s, i, t}\left\{\delta_{t}^{s, i}\right\} \mid(|T|-1) \\
& =6.5+\max \{1.765 ; 1.722 ; 1.685 ; 1.818\}+6.5-1+0.2 \cdot(4-1)=14.418
\end{aligned}
$$

Again, the LP relaxation of the model is solved first. The solution shows that constraint (1.4) is active for all sites in all four periods, indicating that the production is again limited by capacity. Therefore, from equations (11) and (12), we can set the bounds to,

$$
0 \leq \lambda f_{t=1}^{s, m}, \lambda f_{t=2}^{s, m}, \lambda v_{t=1}^{s}, \lambda v_{t=2}^{s} \leq U P_{\text {capacity }}^{\lim }=29.6
$$

Table 4a shows the progress of each algorithm, while Table $4 \mathrm{~b}$ lists the number of cutting plane iterations required for convergence within a 1\% tolerance (gap between bounds). 
Table 4a Progress of cutting plane algorithm with and without bounded multipliers in Example 2

\begin{tabular}{|c|c|c|c|c|c|c|c|c|}
\hline & \multicolumn{4}{|c|}{ Spatial Decomposition } & \multicolumn{3}{c|}{ Temporal Decomposition } \\
\hline Iteration & \multicolumn{2}{|c|}{ Generic Bounds } & \multicolumn{2}{|c|}{ Economic Bounds } & \multicolumn{2}{c|}{ Generic Bounds } & \multicolumn{2}{c|}{ Economic Bounds } \\
\hline & UB [\$] & gap [\%] & UB [\$] & gap [\%] & UB [\$] & gap [\%] & UB [\$] & gap [\%] \\
\hline $\mathbf{1}$ & 67513 & $>>10000$ & 67513 & 153 & 67689 & $>>10000$ & 67689 & 166.7 \\
\hline $\mathbf{5 0}$ & 67513 & $>>10000$ & 67513 & 10.1 & 67689 & 41.1 & 67689 & 16.9 \\
\hline $\mathbf{1 0 0}$ & 67513 & 76.6 & 67513 & 6.3 & 67689 & 14.6 & 67689 & 6.0 \\
\hline $\mathbf{2 0 0}$ & 67513 & 18.3 & 67513 & 2.1 & 67689 & 5.1 & 67689 & 2.5 \\
\hline $\mathbf{5 0 0}$ & 67513 & 7.9 & 67513 & 1.3 & 67600 & 1.4 & - & - \\
\hline $\mathbf{1 0 0 0}$ & 67513 & 4.2 & 67319 & - & - & - & - & - \\
\hline $\mathbf{1 0 0 0 0}$ & - & - & - & - & - & - & - & - \\
\hline
\end{tabular}

Table 4b Convergence of cutting plane algorithm with bounded multipliers in Example 2

\begin{tabular}{|c|c|c|}
\hline & Spatial Decomposition & Temporal Decomposition \\
\hline Iterations Generic Bounds & 2725 & 573 \\
\hline Iterations Economic Bounds & 1308 & 363 \\
\hline Upper bound on profit & $\$ 66957$ & $\$ 66945$ \\
\hline
\end{tabular}

The optimal bounds provided by spatial and temporal decomposition were very similar in this example and within $0.22 \%$ and $0.20 \%$ of global optimality $(\$ 66,810 / 4-$ monthperiod) respectively.

Table 4 reinforces the two observations made in the first example. Temporal decomposition achieves savings of over $70 \%$ of the iterations needed to reach convergence. Furthermore, the economic bounds further reduce the numbers of iterations by over $35 \%$ for temporal and by over $50 \%$ for spatial decomposition.

\section{Example 3}

This example is included with the objective of testing the effectiveness of both decomposition techniques relative to the full space solution of the MILP planning problem. The problem consists of six production sites, six markets, six products, and six time periods. If the six products are thought of as families of products, then the problem 
can be said to be a realistic representation of a small manufacturing network. The MILP formulation consists of 216 discrete variables, 7,957 continuous variables, and 6,985 constraints. The problem size is moderate, but the constraints used to model sequencedependent changeovers make its solution computationally challenging. The sequencing constraints correspond to those in the single-site aggregate planning model by ErdirikDogan \& Grossmann (2008), and are shown in the Appendix A. The sequencing model is based on the traveling salesman problem, which is known to be NP-hard.

We solve the temporal and spatial duals using the subgradient algorithm, as presented in Appendix B and illustrated in Figure 8. The tunable parameter $\varepsilon_{k}$ is set to 0.1 . We initialize the Lagrange multipliers by solving the LP relaxation of the full space problem and assigning the optimal dual variables of constraints (1.14), (1.15) and (1.16) to $\lambda v, \lambda t r$, and $\lambda f$. The subgradient algorithm requires an estimate of the optimal dual solution. We propose two alternatives to obtain this estimate. The first alternative is to run the full space model using an efficient MILP solver, like CPLEX, to generate a feasible solution. This strategy is useful when good feasible solutions are available early on the Branch and Bound algorithm. For this reason we set a short time limit for this initialization step. The second alternative can be used when the full space model is too large and/or a feasible solution is not available in a reasonable time. If this is the case, we propose to use a simple heuristic that consists in fixing the value of the binary variables to those obtained in the solution to the Lagrangean subproblems, $T L_{\lambda}$ in temporal decomposition, or $S L_{\lambda}$ in the spatial decomposition. There is no guarantee that the solution will be feasible, although in our calculations we were always able to construct a feasible solution in this way. Since the subgradient is an iterative algorithm, we solve $T L_{\lambda}$ or $S L_{\lambda}$, and we construct a heuristic solution in every iteration. The solution to the Lagrangean subproblem is an upper bound, and the heuristic solution is a lower bound that is also used for estimating the optimal dual solution in the subgradient algorithm. The upper and lower bounds are only updated in iterations were there is improvement, i.e., when we find tighter bounds.

We solve the temporally and spatially decomposed problems with and without the economic bounds for the multipliers. The problem is set up in a way that monthly market 
demand is sometimes greater and sometimes smaller than production capacity. Therefore, there is incentive to overproduce and build up inventory in those months were there is extra capacity. All calculations were performed using the MILP solver CPLEX 12.1 with GAMS 23.3.3, and run using a $2.79 \mathrm{GHz}$ processor on machine with $2.5 \mathrm{~GB}$ of RAM.

In this example we were able to obtain a good feasible solution within $600 \mathrm{CPU}$ seconds using CPLEX. Table 5 shows the iterations of the algorithm and the gap that corresponds to using the feasible solution obtained with the full space model as a lower bound. When generating the results in this table we do not enforce any bounds on the multipliers.

Table 5: Temporal and spatial decomposition results with unbounded multipliers

\begin{tabular}{|c|c|c|c|c|c|c|c|c|c|}
\hline \multicolumn{5}{|c|}{ Temporal Decomposition } & \multicolumn{5}{|c|}{ Spatial Decomposition } \\
\hline CPU s & Iteration & UB & LB* & gap \% & CPU s & Iteration & UB & LB* & gap \% \\
\hline 711 & 1 & 48032.69 & 47393.5 & 1.35 & 640 & 1 & 48444.42 & 47393.5 & 2.22 \\
\hline 834 & 2 & 48032.17 & 47393.5 & 1.35 & 714 & 2 & 48444.42 & 47393.5 & 2.22 \\
\hline 1378 & 5 & 48026.19 & 47393.5 & 1.33 & 927 & 5 & 48393.57 & 47393.5 & 2.11 \\
\hline 2153 & 10 & 48024.98 & 47393.5 & 1.33 & 1352 & 10 & 48362.68 & 47393.5 & 2.04 \\
\hline 2890 & 15 & 48011.29 & 47393.5 & 1.30 & 1808 & 15 & 48343.9 & 47393.5 & 2.01 \\
\hline 3592 & 20 & 48007.47 & 47393.5 & 1.30 & 2324 & 20 & 48319.1 & 47393.5 & 1.95 \\
\hline
\end{tabular}

Figure 10 shows a plot of the first 3,000 seconds of computation time versus the value of the upper bound obtained by temporal and spatial decomposition. After 3,000 seconds, the temporal decomposition is in iteration 15, while the spatial decomposition is done with the 20 iterations that were set as maximum in the algorithm. From this observation we can infer that temporal decomposition yields tighter bounds, but that the subproblems are more expensive to solve than in spatial decomposition. The five remaining iterations in temporal decomposition do not improve the solution any further. This figure includes the upper and lower bounds obtained by CPLEX using the full space model, after 600 seconds and 4 hours of computation. Notice that the points corresponding to 4 hours do not correspond to their placement with respect to the time axis in Figure 10. 


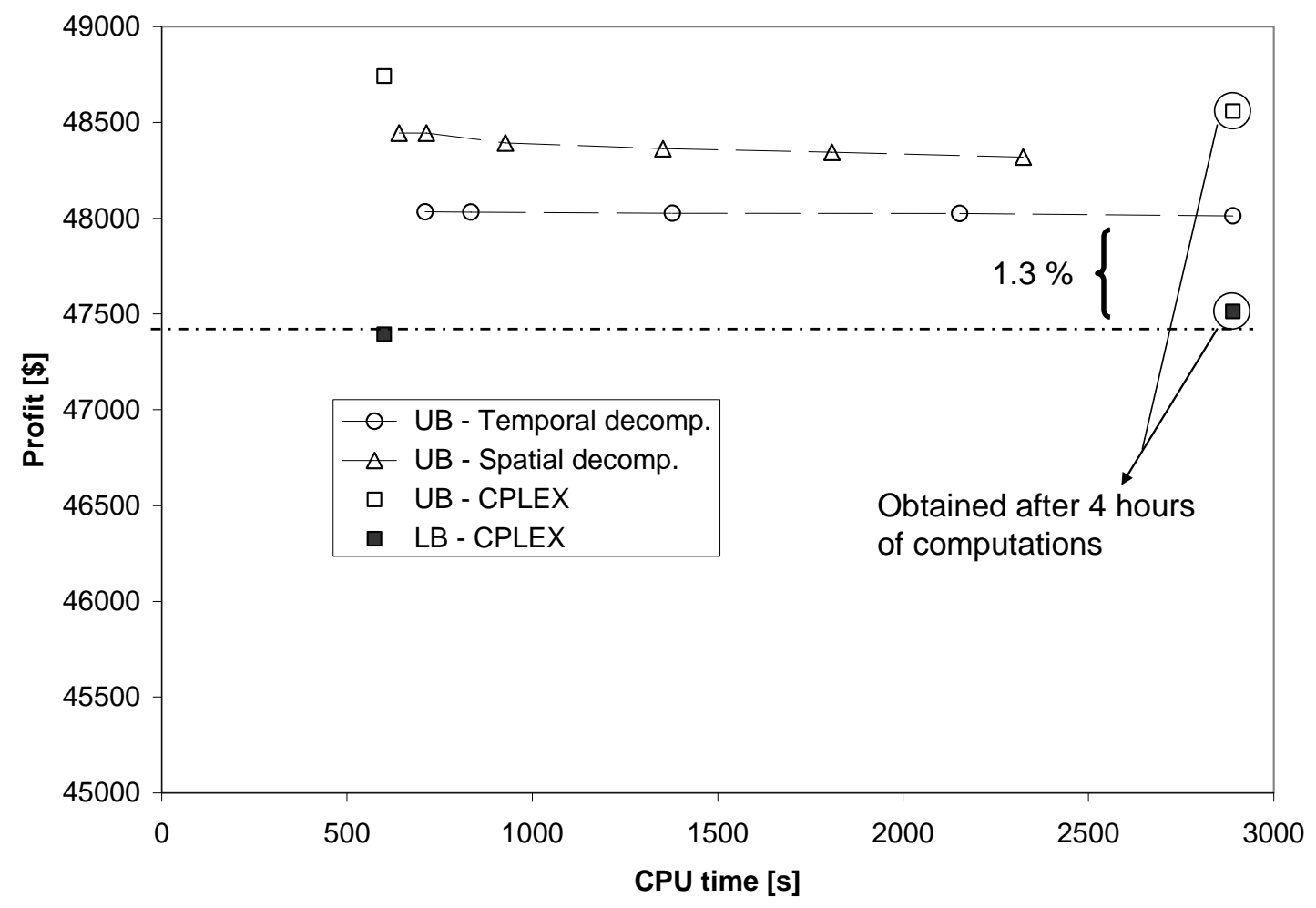

Fig.10 Computational time versus bounds obtained with different solution alternatives

We can make the following observations from the data in Table 5 and Figure 10.

- The bound obtained using temporal decomposition is tighter than the bound obtained using spatial decomposition.

- The upper bound and the gap between the bounds is better for temporal and spatial decomposition than those obtained by CPLEX using the full space model

- A tight bound $(\sim 1.3 \%)$ is obtained by using the heuristics in CPLEX for $600 \mathrm{CPU}$ seconds and then getting an upper bound from the first iteration of temporal decomposition.

The performance of the algorithm when the economic bounds are enforced on the multipliers is not improved significantly in this example, with neither of the two decomposition schemes. The reason is that in this example we have a good starting point when using the lower bound form CPLEX and the values of the multipliers of the dualized constraints from the linear relaxation of the model. In this case the subgradient method is unlike to overstep, and the multiplier bounds are usually not active. 


\section{Example 4}

The last example is included in order to test the efficiency of our algorithm with a larger problem. This instance of the planning problem includes 20 products, 10 markets, 6 production sites, and 6 time periods. The resulting MILP has 720 discrete variables, 68,437 continuous variables, and 52,381 constraints. Example 4 is handled in the same way as Example 3 with regards to the tunable parameter $\varepsilon_{k}$ and to the initialization of the Lagrange multipliers. In this example we cannot obtain a feasible solution from CPLEX in a short enough time (it takes as much as running several iterations of the decomposition algorithm) so we construct feasible solutions by the second alternative described in Example 3. That is, we fix the value of the binary variables to those obtained in the solution to Lagrangean sub problems, $T L_{\lambda}$ in temporal decomposition, or $S L_{\lambda}$ in spatial decomposition.

The results are summarized in Figure 11, where it can be seen that temporal decomposition provides tighter bounds in shorter computation time. No significant improvement is reported for the temporal decomposition when the economic bounds are enforced. In contrast the progress of the algorithm using spatial decomposition was enhanced by bounding the multipliers. We think that since spatial decomposition has a looser relaxation, the beneficial effect of bounding the multipliers has a more significant effect than in temporal decomposition. Also, we do not derive any bounds on the multipliers of constraint (1.14), which are involved in temporal decomposition, whereas we derive bounds for all the multipliers in spatial decomposition. 


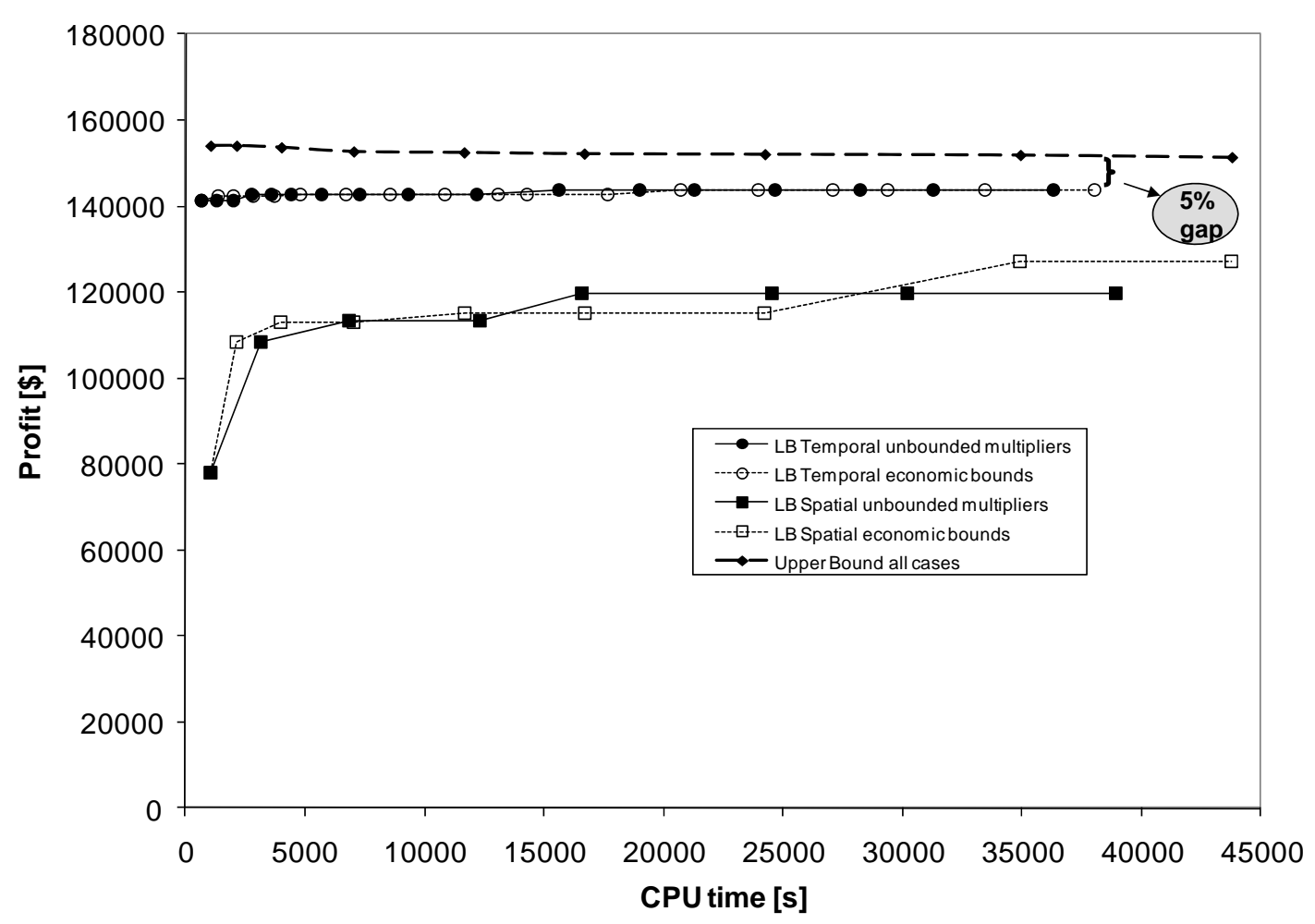

Fig. 11 Performance of temporal and spatial decompositions with and without enforcing multiplier bounds in Example 2

\section{Conclusions}

We have presented a theoretical result that explains the observation made in previous works that temporal decomposition provides tighter bounds than spatial decomposition in some types of production planning problems. We have presented the theoretical result in the context of a multi-site, multi-period, multi-product planning problem with sequencedependent changeovers. The changeovers that we considered are modeled using sequencing constraints that yield a mixed-integer linear programming (MILP) problem. Our result indicate that the optimal duality gap in temporal decomposition will always be at least as small as the one obtained using spatial decomposition.

In the second part of this paper we have proposed a method to obtain bounds on the optimal value of the Lagrange multipliers of temporal and spatial decomposition. The technique is based on an economic interpretation of the Lagrange multipliers from duality theory. 
We have presented numerical results for four examples. The first two are used to give some numerical evidence that the optimal solution of the temporal dual is at least as tight as in the spatial dual. The cutting plane algorithm was used to solve these two examples. Applying the economic bounds on the multipliers speeds the convergence of this algorithm, particularly when solving the spatial dual. The third and fourth examples consist of larger planning problems and were solved using the subgradient method. The results indicate that the decomposition strategies are efficient for larger problems, and that the bound provided by temporal decomposition was confirmed to be tighter than the bound from spatial decomposition. The economic bounds enhanced the convergence of spatial decomposition in larger problems with loose initial bounds.

\section{Acknowledgments}

The authors would like to acknowledge financial support by the Dow Chemical Company for this project.

\section{References}

Chen P. and J. M. Pinto (2008). Lagrangean-based techniques for the supply chain management of flexible process networks. Computers and Chemical Engineering, 32, $2505-2528$.

Chvátal V., Linear Programming. W. H. Freeman and Company, New York (1983).

Dorfman R., P. A. Samuelson and R. M. Solow, Linear Programming and Economic Analysis. McGraw Hill Book Company, New York (1958).

Erdirik-Dogan M. and I. E. Grossmann (2008). Simultaneous planning and scheduling of single-stage multiproduct continuous plants with parallel lines. Computers and Chemical Engineering, 32, 2664 - 2683.

Guignard M. (2003). Lagrangean Relaxation. Top, 11(2), 151 - 228 .

Geoffrion A. M. (1974). Lagrangean Relaxation for Integer Programming. Mathematical Programming Study, 2, 82 - 114.

Gupta A. and C. D. Maranas (1999). A Hierarchical Lagrangean Relaxation Procedure for Solving Midterm Planning Problems. Industrial and Engineering Chemistry Research, 38, 1937 - 1947. 
Jackson J. R. and I. E. Grossmann (2003). Temporal Decomposition Scheme for Nonlinear-Multisite Production Planning and Distribution Models. Industrial and Engineering Chemistry Research, 42(13), 3045 - 3055.

Neiro S.M.S. and J. M. Pinto (2006). Lagrangean decomposition applied to multiperiod planning of petroleum refineries under uncertainty. Latin American Applied Research, $36,213-220$.

Pochet Y. and L. A. Wolsey. Production Planning by Mixed Integer Programming. Springer, New York (2006).

Trotter P. A.,(2009). Economic Interpretation of Lagrange Multipliers in Lagrangean Decomposition of a Planning Problem, Technical Report, RWTH Aachen University.

Williams H. P. (1996). Duality in Mathematics and Linear and Integer Programming. Journal of Optimization Theory and Applications, 90(2), 257 - 278.

Wu D. and M. Ierapetritou (2006). Lagrangean decomposition using an improved NelderMead approach for Lagrangean multiplier update. Computers and Chemical Engineering, 30, $778-789$.

\section{Appendix A}

\section{Production planning model}

The model presented in this appendix is an extension of the MILP model by ErdirikDogan and Grossmann (2008) for the case of multi-site production of continuous multiproduct plants with parallel lines.

Nomenclature

Index/Set

i,k/I indices/set of products

$s / S \quad$ index/set of manufacturing sites

$t / T \quad$ index/set of time periods

$m / M \quad$ index/set of markets

Parameters 
$\beta_{t}^{m, i}$

$\alpha_{t}^{s, i}$

$\delta_{t}^{s, i}$

$\gamma_{t}^{s, m, i}$

$T C_{t}^{s, i, k}$

$a_{t}^{s, i}$

$b s_{t}^{s, i}$

$b t_{t}^{s, i, k}$

$x_{s, i}^{U B}$

$v_{s, i}^{U P}$

$f_{s, i}^{U P}$

$d_{t}^{m, i}$

$L_{t}$

Variables

$s l_{t}^{s, m, i}$

$x_{t}^{s, i}$

$v_{t}^{s, i}$

$s l_{t}^{s, m, i}$

$X F_{i, s, t}$

$X L_{i, s, t}$

$\operatorname{setup}_{i, s, t}$ sale price of product $i$ in market $m$ during time period $t$

production cost of product $i$ in production site $s$ during time period $t$

inventory cost of product $i$ in production site $s$ during time period $t$

shipment cost between production site $s$ and market $m$

cost incurred during a transition from product $i$ to product $k$

production rate for product $i$ in production site $s$ during time period $t$

time for setting up the production of product $i$ in production site $s$

length of a transition from product $i$ to product $k$

upper bound on production of product $i$ in production site $s$

maximum storage capacity for product $i$ in production site $s$

maximum shipping amount between production site $s$ and market $m$

demand for product $i$ in market $m$ during time period $t$

Length of time period $t$

sales of product $i$ manufactured in site $s$ and sold in market $m$

production of product $i$ in manufacturing site $s$ during time period $t$

inventory of product $i$ in site $s$ at the end of time period $t$

shipment of product $i$ from manufacturing site $s$ to market $m$

continuous variable bounded at 0 and 1 to denote if product $i$ is the first product in site $s$ during period $t$

continuous variable bounded at 0 and 1 to denote if product $i$ is the last product in site $s$ during period $\mathrm{i}$

$0-1$ variable to denote if product $i$ is assigned to site $s$ during time period 
$Z P_{i, k, s, t}$

continuous variable bounded at 0 and 1 to denote if product $i$ precedes product $k$ in unit $\mathrm{m}$ during time period $t$

$Z Z P_{i, k, s, t} \quad$ continuous variable bounded at 0 and 1 to denote if the link between products $i$ and $k$ are broken

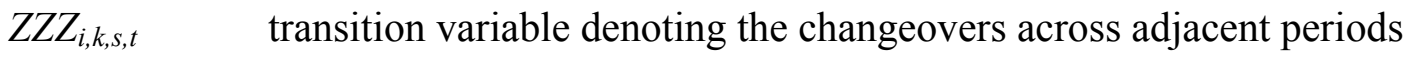

\section{Constraints}

$$
\begin{aligned}
\max \pi= & \sum_{t \in T} \sum_{s \in S} \sum_{m \in M} \sum_{i \in I} \beta_{t}^{m, i} s l_{t}^{s, m, i}-\sum_{t \in T} \sum_{s \in s} \sum_{i \in I}\left(\alpha_{t}^{s, i} x_{t}^{s, i}+\delta_{t}^{s, i} v_{t}^{s, i}\right)- \\
& \sum_{t \in T} \sum_{s \in S} \sum_{m \in M} \sum_{i \in I} \gamma_{t}^{s, m, i} f_{t}^{s, m, i}-\sum_{t \in T} \sum_{s \in S i \in I} \sum_{k \in I} T C_{t}^{s, i, k}\left(Z P_{i, k, m, t}+Z Z Z_{i, k, m, t}-Z Z P_{i, k, m, t}\right)
\end{aligned}
$$

s.t.

$v_{t-1}^{s, i}+x_{t}^{s, i}=\sum_{m \in M} f_{t}^{s, m, i}+v_{t}^{s, i} \quad i \in I, s \in S, t \in T$

$x_{t}^{s, i} \leq x_{s, i}^{U B} \operatorname{stp}_{t}^{s, i} \quad i \in I, s \in S, t \in T$

$\sum_{i \in I}\left(a_{t}^{s, i} x_{t}^{s, i}+b s_{t}^{s, i} s t p_{t}^{s, i}+\sum_{k \in I} b t_{t}^{s, i, k}\left(Z P_{i, k, m, t}+Z Z Z_{i, k, m, t}-Z Z P_{i, k, m, t}\right)\right) \leq L_{t}$

$f_{t}^{s, m, i}=s l_{t}^{s, m, i}$

$s \in S, t \in T$

$\sum_{s \in S} s l_{t}^{s, m, i} \leq d_{t}^{m, i}$

$i \in I, s \in S, m \in M, t \in T$

$v_{t}^{s, i} \leq v_{s, i}^{U B}$

$i \in I, m \in M, t \in T$

$f_{t}^{s, m, i} \leq f_{s, m, i}^{U B}$

$i \in I, s \in S, t \in T$

$\operatorname{setup}_{i, s, t}=\sum_{k \in I} Z P_{i, k, s, t}$

$i \in I, s \in S, m \in M, t \in T$

$\operatorname{setup}_{k, s, t}=\sum_{k \in I} Z P_{i, k, s, t}$

$i \in I, s \in S, t \in T$

$\sum_{i \in I} \sum_{k \in K} Z Z P_{i, k, m, t}=1$

$k \in I, s \in S, t \in T$

$Z Z P_{i, k, m, t} \leq Z P_{i, k, m, t}$

$s \in S, t \in T$

$\operatorname{setup}_{i, s, t} \geq Z P_{i, i, s, t}$

$i \in I, k \in I, s \in S, t \in T$

$Z P_{i, i, s, t}+\operatorname{setup}_{k, s, t} \leq 1$

$i \in I, s \in S, t \in T$

$Z P_{i, i, s, t} \geq \operatorname{setup}_{i, s, t}-\sum_{k \neq i, k \in I} \operatorname{setup}_{k . m . t} \quad i \in I, s \in S, t \in T$ 


$$
\begin{array}{lr}
X F_{k, s, t} \geq \sum_{i \in I} Z Z P_{i, k, s, t} & i \in I, s \in S, t \in T \\
X L_{i, s, t} \geq \sum_{k \in I} Z Z P_{i, k, s, t} & i \in I, s \in S, t \in T \\
\sum_{i \in I} X F_{i, s, t}=1 & i \in I, s \in S, t \in T \\
\sum_{i \in I} X L_{i, s, t}=1 & i \in I, s \in S, t \in T \\
\sum_{k \in I} Z Z Z_{i, k, s, t}=X L_{i, s, t} & i \in I, s \in S, t \in T \\
\sum_{i \in I} Z Z Z_{i, k, s, t}=X F_{k, s, t+1} & k \in I, s \in S, t \in T
\end{array}
$$

\section{Appendix B}

\section{Methods for solving the Lagrangean Dual (D)}

In this section, we give an overview of the cutting plane and the subgradient methods. Following the notation of Guignard (2003), we define the Lagrangean function as $z(\lambda)=v\left(L R_{\lambda}\right)$. If the polytope $\operatorname{Co}\{x \in X \mid C x \leq d\}$ is represented by its extreme points $\left\{x^{1}, x^{2}, \ldots, x^{k}\right\}$, then $z(\lambda)=\min _{k=1, \ldots K}\left\{f x^{k}+\lambda\left(A x^{k}-b\right)\right\}$. Thus, $z(\lambda)$ is a lower envelope of piecewise-linear functions of $\lambda$.

The cutting plane method solves the Lagrangean dual $\max _{\lambda \geq 0} v\left(L R_{\lambda}\right)=\max _{\lambda \geq 0} z(\lambda)$ by adding one constraint of the type $f x^{k}+\lambda\left(A x^{k}-b\right)$ at a time, so that by iteration $k$ we have $h=1, \ldots, k$ such constraints. The point $x^{k}$ added after every iteration corresponds to the solution of the Lagrangean subproblem $\left(L R_{\lambda}\right)$. The accumulated cuts are used to define the following LP master problem:

$$
\begin{aligned}
& \max _{\lambda \geq 0} z(\lambda)=\max _{\lambda \geq 0} \min _{h=1, \ldots k}\left\{f x^{h}+\lambda\left(A x^{h}-b\right)\right\}= \\
& \max _{\lambda \geq 0, \eta}\left\{\eta \mid \eta \leq f x^{h}+\lambda\left(A x^{h}-b\right), h=1, \ldots k\right\}
\end{aligned}
$$


The solution to $\left(M P^{k}\right)$ is used as the next $\lambda$ in $z\left(\lambda^{k+1}\right)$. The algorithm terminates when $v\left(M P^{k}\right)=z\left(\lambda^{k+1}\right)$.

The subgradient method is an iterative algorithm that, given a vector of Lagrange multipliers $\lambda^{k}$, obtains the vector $\lambda^{k+1}$ by stepping along the subgradient of $z\left(\lambda^{k}\right)$. Let $x^{k}$ be an optimal solution of $\left(L R_{\lambda}\right)$ with $\lambda^{k}$. Then the subgradient of $z\left(\lambda^{k}\right)$ is $s^{k}=\left(A x^{k}-b\right)^{T}$. The formula for updating the vector of Lagrange multipliers using subgradient information is as follows,

$$
\lambda^{k+1}=\lambda^{k}+\frac{s^{k} \varepsilon_{k}\left(z^{*}-z\left(\lambda^{k}\right)\right)}{\left\|s^{k}\right\|^{2}} .
$$

In this formula $z^{*}$ is an approximation of the optimal Lagrangean dual solution. The term $\varepsilon_{k} \in[0,2]$ is used to correct the over/under estimation of the true optimal dual solution. The precise value of $\varepsilon_{k}$ is selected using heuristic rules. Once $\lambda^{k+1}$ is obtained $\left(L R_{\lambda}\right)$ is solved again until a stopping criterion is met or the maximum number of iterations is reached. In practice the most widely used stopping criteria are the magnitude of the improvement of $\left(L R_{\lambda}\right)$ in successive iterations and the norm of the subgradient $\left\|s^{k}\right\|^{2}$.

\section{Appendix C}

The simplified production model used in example one

The following MILP model corresponds to the planning problem used in example one which does not take sequencing into consideration. Therefore, constraint (1.5) is dropped and the transition cost $T C_{t}^{s}$ becomes a cost that has to be paid for production setup. Constraint (C.3) ensures that production requires paying these setup costs. The copy variables that are necessary to decompose the problem are already introduced. 


$$
\begin{array}{ll}
\max \pi=\sum_{t \in T} \sum_{s \in S} \sum_{m \in M} \beta_{t}^{m} s l_{t}^{s, m}-\sum_{t \in T} \sum_{s \in s}\left(\alpha_{t}^{s} x_{t}^{s}+\delta_{t}^{s} v_{t}^{s}+T C_{t}^{s} s t p_{t}^{s}\right)-\sum_{t \in T} \sum_{s \in S} \sum_{m \in M} \gamma_{t}^{s, m} \bar{f}_{t}^{s, m} \\
\text { s.t. } & \forall t \in T, s \in S \\
\bar{v}_{t-1}+x_{t}^{s}=\sum_{m \in M} f_{t}^{s, m}+v_{t}^{s} & \\
x_{t}^{s} \leq x_{s}^{U B} s t p_{t}^{s} & \forall t \in T, s \in S \\
\mathrm{a}_{\mathrm{t}}^{\mathrm{s}} \mathrm{x}_{\mathrm{t}}^{\mathrm{s}}+\mathrm{bs}_{\mathrm{t}}^{\mathrm{s}} \mathrm{stp}_{\mathrm{t}}^{\mathrm{s}} \leq \mathrm{L}_{\mathrm{t}} & \forall t \in T, s \in S \\
\bar{f}_{t}^{s, m}=s l_{t}^{s, m} & \forall t \in T, s \in S, m \in M \\
\sum_{s \in S} s l_{t}^{s, m} \leq d_{t}^{m} & \forall t \in T, m \in M \\
x_{t}^{s} \leq x_{s}^{U B} ; v_{t}^{s} \leq v_{s}^{U B}, v_{t}^{s} \leq v_{s}^{U B} & \forall t \in T, s \in S \\
f_{t}^{s, m} \leq f_{s, m}^{U B}, \bar{f}_{t}^{s, m} \leq f_{s, m}^{U B} & \forall t \in T, s \in S, m \in M \\
\mathrm{x}_{\mathrm{t}}^{\mathrm{s}} \in \mathfrak{R}_{+}^{\mid \mathrm{II}}, \mathrm{v}_{\mathrm{t}}^{\mathrm{s}} \in \mathfrak{R}_{+}^{|I|} & \forall t \in T, s \in S \\
\overline{\mathrm{V}}_{\mathrm{s}}^{\mathrm{s}} \in \mathfrak{R}_{+}^{\mid \mathrm{II}} & \forall t \in T, s \in S \\
f_{t}^{s, m} \in \mathfrak{R}_{+}^{|I|}, \bar{f}_{t}^{s, m} \in \mathfrak{R}_{+}^{|I|}, s l_{t}^{s, m} \in \mathfrak{R}_{+}^{|I|} & \forall t \in T, s \in S, m \in M \\
s t p_{t}^{s} \in\{0,1\}^{|I|} & \forall t \in T, s \in S \\
\bar{v}_{t}^{s}=v_{t}^{s} & \forall t \in T, s \in S \\
\bar{f}_{t}^{s, m}=f_{t}^{s, m} & \forall t \in T, s \in S
\end{array}
$$

